\title{
Susceptibility to diet induced obesity at thermoneutral conditions is independent of UCP1
}

Sebastian Dieckmann ${ }^{1,2,3}$, Akim Strohmeyer ${ }^{1,2,3}$, Monja Willershäuser ${ }^{1,2,3}$, Stefanie Maurer ${ }^{1,2,3}$, Wolfgang Wurst ${ }^{4,5,6}$, Susan Marschall ${ }^{7}$, Martin Hrabe de Angelis ${ }^{7,8,9}$, Ralf Kühn ${ }^{10}$, Anna Worthmann ${ }^{11}$, Marceline M Fuh ${ }^{11}$, Joerg Heeren ${ }^{11}$, Nikolai Köhler ${ }^{12}$, Josch K. Pauling ${ }^{12}$, Martin Klingenspor ${ }^{1,2,3, *}$

${ }^{1}$ Chair for Molecular Nutritional Medicine, Technical University of Munich, TUM School of Life Sciences, Freising, Germany.

${ }^{2}$ EKFZ - Else Kröner-Fresenius Center for Nutritional Medicine, Technical University of Munich, Freising, Germany.

${ }^{3}$ ZIEL - Institute for Food \& Health, Technical University of Munich, Freising, Germany.

${ }^{4}$ Institute of Developmental Genetics, Helmholtz Zentrum München, Germany.

${ }^{5}$ Technische Universität München-Weihenstephan 85764 Neuherberg/Munich, Germany.

${ }^{6}$ German Center for Neurodegenerative Diseases (DZNE), Site Munich, Germany.

${ }^{7}$ Institute of Experimental Genetics, Helmholtz Zentrum München, Germany.

${ }^{8}$ Chair of Experimental Genetics, TUM School of Life Sciences, Technical University of Munich, Freising, Germany.

${ }^{9}$ German Center for Diabetes Research (DZD), Neuherberg, Germany.

${ }^{10}$ Institute of Developmental Genetics, Helmholtz Zentrum München, Germany. Present address: MaxDelbrück-Center for Molecular Medicine in the Helmholtz Association, Berlin, Germany.

${ }^{11}$ Department of Biochemistry and Molecular Cell Biology, University Medical Center HamburgEppendorf, Hamburg, Germany.

${ }^{12}$ LipiTUM, Chair of Experimental Bioinformatics, TUM School of Life Sciences, Technical University of Munich, Freising, Germany.

*Corresponding Author:

Prof. Dr. Martin Klingenspor

Chair of Molecular Nutritional Medicine

TUM School of Life Sciences Weihenstephan

Technical University of Munich

Gregor-Mendel-Str.2

D-85354 Freising, Germany

Tel: $\quad+49(0) 8161712386$

Fax: +49 (0)8161 712404

Email: mk@tum.de 


\section{Abstract}

\section{Objective}

Activation of uncoupling protein 1 (UCP1) in brown adipose tissue (BAT) upon cold stimulation leads to substantial increase in energy expenditure to defend body temperature. Increases in energy expenditure after a high caloric food intake, termed diet-induced thermogenesis, are also attributed to BAT. These properties render BAT a potential target to combat diet-induced obesity. However, studies investigating the role of UCP1 to protect against diet-induced obesity are controversial and rely on the phenotyping of a single constitutive UCP1-knockout model.

To address this issue, we generated a novel UCP1-knockout model by Cre-mediated deletion of Exon 2 in the UCP1 gene. We studied the effect of constitutive UCP1 knockout on metabolism and the development of diet-induced obesity.

\section{Methods}

UCP1 knockout and wildtype mice were housed at $30^{\circ} \mathrm{C}$ and fed a control diet for 4-weeks followed by 8-weeks of high-fat diet. Body weight and food intake were monitored continuously over the course of the study and indirect calorimetry was used to determine energy expenditure during both feeding periods.

\section{Results}

Based on Western blot analysis, thermal imaging and noradrenaline test, we confirmed the lack of functional UCP1 in knockout mice. However, body weight gain, food intake and energy expenditure were not affected by deletion of UCP1 gene function during both feeding periods.

\section{Conclusion}

Conclusively, we show that UCP1 does not protect against diet-induced obesity at thermoneutrality. Further we introduce a novel UCP1-KO mouse enabling the generation of conditional UCP1-knockout mice to scrutinize the contribution of UCP1 to energy metabolism in different cell types or life stages. 


\section{Introduction}

Thermogenic brown adipose tissue (BAT) is the main contributor to non-shivering thermogenesis, the process to maintain normothermia in a variety of small mammals. Non-shivering thermogenesis is mediated by the uncoupling protein 1 (UCP1), which enables high rates of oxygen consumption by the mitochondrial electron transport chain without ATP production. The most potent stimulation of UCP1 and non-shivering thermogenesis is mediated by the sympathetic innervation of BAT with the neurotransmitter norepinephrine triggering beta-3-adrenergic receptor signaling in brown adipocytes. Furthermore, meal-associated thermogenesis in BAT is activated upon food intake (Glick, Teague, and Bray 1981) by the prandial surge of the gut peptide hormone secretin (Li et al. 2018). Other activators of BAT thermogenesis have been reported (Zietak and Kozak 2016; Gnad et al. 2014) and recent findings suggest that brown fat conveys effects on systemic metabolism and energy balance by means of paracrine intercellular and endocrine interorgan crosstalk. The beneficial metabolic effects of BAT clearly go beyond the combustion of calories (Kajimura, Spiegelman, and Seale 2015). Activation of meal-associated thermogenesis in BAT initiates meal termination and thereby contributes the control of energy intake (Schnabl, Li, and Klingenspor 2020). Together with the potential of BAT to impact energy balance and systemic metabolism by clearing glucose from circulation, these characteristics render UCP1 and BAT potential targets to improve cardiometabolic health and the treatment of type 2 diabetes (Becher et al. 2021).

In this context the question whether UCP1 can protect against diet induced obesity (DIO) has been studied repeatedly. Standard housing temperature $\left(20-23^{\circ} \mathrm{C}\right)$ represents a mild cold challenge for laboratory mice resulting in a two-fold increase of daily energy expenditure (Fischer, Cannon, and Nedergaard 2018). BAT is the source for this thermoregulatory heat production. UCP1 knockout mice when kept at standard housing temperature do not develop diet-induced obesity (Bond and Ntambi 2018) and even seem to be protected, having lower body weight than WT mice (Liu et al. 2003; T. Wang et al. 2008; Keipert et al. 2020). It has been proposed that this is due to alternative thermogenic mechanisms less efficient than UCP1-dependent thermogenesis (Liu et al. 2003; T. Wang et al. 2008; Keipert et al. 2020), that are recruited to cope with the need for thermoregulatory heat production in UCP1-KO mice. 
Housing mice at higher temperatures $\left(27^{\circ} \mathrm{C}-30^{\circ} \mathrm{C}\right)$, corresponding to their thermoneutral zone eliminates this heat sink. Since UCP1 in BAT is inactive at thermoneutrality, the lack of thermogenic BAT function should be without consequences for energy balance. Indeed, several studies using the established UCP1-KO mouse model originally generated by Leslie Kozak and coworkers confirmed this expectation (Enerbäck et al. 1997; Liu et al. 2003; Zietak and Kozak 2016; Winn et al. 2017; Maurer et al. 2020; Fischer et al. 2020). In contrast, other studies reported that UCP1 knockout mice are more susceptible to diet induced obesity at thermoneutrality (Feldmann et al. 2009; von Essen et al. 2017; Rowland et al. 2016; Luijten et al. 2019; Pahlavani et al. 2019). One explanation for the increased susceptibility to DIO in UCP1-KO mice may be adaptations in metabolism, leading to a more efficient metabolism or the lack of diet-induced thermogenesis in UCP1-KO mice (von Essen et al. 2017). However, recent data from a UCP1 knockdown model (H. Wang et al. 2021) demonstrate that UCP1 abundance alone does not protect against DIO at thermoneutrality. Despite having remarkable reduced but still activatable UCP1 levels, these mice are not more or less prone to DIO compared to wildtype littermates with normal functional levels of UCP1.

This showcases the urgent need for new UCP1-KO models to scrutinize the role of UCP1 on energy balance and metabolism. So far two UCP1 knockout models are available (Enerbäck et al. 1997; Bond and Ntambi 2018). Other transgenic mice with impaired UCP1 expression include knockdown models (Chen, Hsu, and Huang 2018; H. Wang et al. 2019) or diphtheria toxin chain A induced depletion of UCP1 expressing cells (Lowell et al. 1993; Rosenwald et al. 2013).

In the present study we therefore introduce and validate a novel Cre-mediated UCP1-KO model and demonstrate that deletion of UCP1 has no effect on energy balance regulation at thermoneutrality. 


\section{Material and Methods}

\subsection{Animal model}

The UCP1 knockout mouse line was generated in frame of the EUCOMM program and is a constitutive UCP1 knockout model on a C57BL/6N background (Skarnes et al. 2011; Pettitt et al. 2009). It originates from the UCP1 ${ }^{\text {tmla }}$ mouse, carrying a lacZ \& neomycin cassette, two FLP sites and three loxP sites (Figure $1 \mathrm{~A})$. Through crossing with a FLP mouse, the lacZ and neomycin cassette as well as one FLP site and one loxP site are removed, resulting in a UCP1 ${ }^{\text {tmlc }}$ (UCP1-WT) mouse. Cross breeding this mouse with a Rosa26-CRE mouse results in the UCP1 $1^{\text {tmld }}$ (UCP1-KO) mouse carrying a germline deletion of the exon 2 of the UCP1 gene. UCP1-KO and UCP1-WT mice were crossed to generate UCP1-HET mice. The UCP1 knockout line, is maintained by crossing male and female UCP1-HET mice. All studied mice were derived of our heterozygous maintenance breeding. Mice were bred and housed at $23^{\circ} \mathrm{C}$ ambient temperature with a $12 / 12 \mathrm{~h}$ light/dark cycle and had libitum access to water and chow diet.

All animal experiments were performed according to the German animal welfare law and approved by the district government of Upper Bavaria (Regierung von Oberbayern, reference number ROB-55.22532.Vet_02-15-128).

\subsubsection{HFD feeding at thermoneutrality}

Male wildtype $(\mathrm{n}=7)$ and knockout $(\mathrm{n}=7)$ mice for the high fat diet feeding experiment were obtained from our heterozygous maintenance breeding. At the age of 8 weeks, mice were switched from chow to a chemically defined control diet with a fat content of $50 \mathrm{~g} / \mathrm{kg}(\mathrm{CD}, \sim 13 \mathrm{~kJ} \%$ from Fat, $15.3 \mathrm{MJ} / \mathrm{kg}$, Snifff Cat. No S5745-E702). Simultaneously, mice were single caged and transferred to climate cabinets with an ambient temperature of $30{ }^{\circ} \mathrm{C}$ and $55 \% \mathrm{RH}$. After an acclimatization phase of 4 weeks, mice were switched from CD to a high fat diet with a fat content of $250 \mathrm{~g} / \mathrm{kg}$ (HFD, $48 \mathrm{~kJ} \%$ from fat, 19.6 $\mathrm{MJ} / \mathrm{kg}$, Snifff Cat. No S5745-E712). After 8 weeks of HFD feeding, mice were killed by $\mathrm{CO}_{2}$ asphyxiation. Whole blood was taken by cardiac puncture, collected in lithium heparin-coated tubes (Sarstedt, Nümbrecht/Germany), and centrifuged at $4^{\circ} \mathrm{C}$ for $10 \mathrm{~min}$ with $1500 \mathrm{x}$ g. The plasma 
supernatant was transferred to fresh tubes and snap frozen in liquid nitrogen. Subsequently, cecal content and tissues were dissected, weighed, and immediately snap frozen in liquid nitrogen. Cecal content, tissues and plasma were stored at $-80^{\circ} \mathrm{C}$ until further processing. Body weight and food intake were determined twice a week between 12.00 PM and 4.00 PM. Additionally, body composition was determined every other week by nuclear magnetic resonance spectroscopy (mq7.5, Bruker BioSpin $\mathrm{GmbH}$, Rheinstetten/Germany). Mice were maintained on a 12/12 h light/dark cycle and had ad libitum access to water and the respective diets during the whole experiment. Food was replaced completely twice a week to avoid rancidity of the HFD at $30^{\circ} \mathrm{C}$. Energy expenditure, energy intake, energy excretion and metabolic efficiency of CD and HFD-fed mice were assessed as described below.

\subsection{Indirect calorimetry, basal metabolic rate and noradrenaline tests}

Indirect calorimetry was performed based on an open respirometer system (LabMaster System; TSE Systems, Bad Homburg/Germany) similar to previously described methodology (Maurer et al. 2015). $\mathrm{O}_{2}$ consumption and $\mathrm{CO}_{2}$ production were determined after 2.5 weeks of feeding $\mathrm{CD}$ and after 4 weeks of HFD. Mice were transferred in specially equipped cages in a climate cabinet (KPK 600, Feutron, Germany) set to $30^{\circ} \mathrm{C}$ after determining body weight and body composition in the afternoon $(2.00-$ 5.00 PM). The measurement was started on the next day at 6.00 AM (CD) or 12.00 PM (HFD) and continued two (CD) or three (HFD) dark phases. The air from the cages was extracted over a period of 1 min every 4-6 min. Heat production was calculated according to (Heldmaier 1975) as: $H P[\mathrm{~mW}]=$ $(4.44+1.43 *$ respiratory exchange ratio $) *$ oxygen consumption $[\mathrm{ml} / \mathrm{h}]$

Basal metabolic rate (BMR) was determined immediately after the last night phase of the indirect calorimetry measurement of the HFD period. Mice were deprived of food between $7.00-8.00$ AM for at least 4 hours. BMR was calculated as the mean of the four lowest consecutive heat production measurements during the last 90 minutes of fasting, similar to a previous published method (Fromme et al. 2019). Subsequently, noradrenaline tests were performed between $10.00 \mathrm{AM}-5.00 \mathrm{PM}$ at $26^{\circ} \mathrm{C}$ to avoid noradrenaline induced hypothermia. Noradrenaline $(1 \mathrm{mg} / \mathrm{kg}$, Arterenol®, Sanofi) was injected intraperitoneally. Air was extracted continuously from the cages with a measurement period of $1 \mathrm{~min}$ over $60 \mathrm{~min}$. 


\subsection{Collection of food spillage and faeces}

Embedding material was collected form cages after indirect calorimetry for each mouse separately, to correct food intake for spillage and to determine energy loss by faecal excretion. Material was dried at room temperature under a chemical flow hood for at least 1 week. Subsequently, cage material was fractionated based on size by shaking the material on a sieve shaker (EML 200 Digital Plus T, Haver \& Boecker, Oelde/ Germany) for $5 \mathrm{~min}$ with an interval of $0.5 \mathrm{~min}$ at an amplitude of 1.4 , through sieves with different mesh sizes $(4,3.15,2.5,1.25$ and $1 \mathrm{~mm}$, VWR International GmbH, Darmstadt/Germany). Flowthrough of the $1 \mathrm{~mm}$ sieve was collected in a pan. Each sieve was scanned for spilled food and faeces (the majority of faeces will be present in the $1.25 \mathrm{~mm}$ sieve). If applicable, food and faeces were picked with tweezers and collected for weighing and determination of energy content by bomb calorimetry. Food intake (in grams) during the indirect calorimetry sessions was corrected for the amount of collected food spillage (in grams).

\subsection{Determination of energy content of food and faeces by bomb calorimetry}

The energy content of the diets and the faecal pellets collected during indirect calorimetry was determined with an isoperibolic bomb calorimeter (Model Nr. 6400, Parr Instrument Company, IL/USA). Energy content of the diets was determined on food samples collected at different time points during the experiment $(\mathrm{CD} n=9, \mathrm{HFD} n=10)$. Energy intake was calculated by multiplying the mean energy content of the diets $(\mathrm{kJ} / \mathrm{g})$ with the amount of food intake (in grams).

The collected faeces was weighed and grinded with metal balls for $2.5 \mathrm{~min}$ at $30 \mathrm{~Hz}$ (Tissue Lyser II, Retsch GmbH. Haan/Germany). Grinded faeces was pressed into a pellet, weighed, and subjected to bomb calorimetry. Benzoeic acid ( 0.7 g) was added as combustion aid. Energy lost via faeces was calculated for each mouse by multiplying the total amount of faeces collected (in grams, see 2.4) by the energy content $(\mathrm{kJ} / \mathrm{g})$ determined by bomb calorimetry.

\subsection{Thermal imaging}

Thermal imaging was performed as described previously (Maurer et al. 2015) with 1-3 day old newborn pups. In brief, at least 3 serial pictures were taken of each litter in 6-well cell culture plates (T890 
thermal imager, Testo, Lenzkirch/Germany). Image analysis was performed with the IRSoft Software (version 4.6, Testo, Lenzkirch/Germany) and the temperature above the interscapular BAT deport (interscapular skin surface temperature, iSST) was determined.

\subsection{Genotyping}

Genotyping was performed on earpieces obtained during tagging of the animals. Tissues were lysed (10 $\mathrm{mM}$ TRIS, $50 \mathrm{mM} \mathrm{KCl}, 0.45 \%$ Nonidet P40, $0.45 \%$ Tween-20, $10 \%$ gelatin in $\mathrm{H} 20$ at $\mathrm{pH} 8.3 \%$ with $0.2 \mathrm{mg} / \mathrm{ml}$ Proteinase $\mathrm{K}$ ) for $4 \mathrm{~h}$ at $65^{\circ} \mathrm{C}$ and vigorous shaking. Proteinase $\mathrm{K}$ was inactivated by heating for $10 \mathrm{~min}$ at $95^{\circ} \mathrm{C}$. PCR (denaturation: $5 \mathrm{~min} / 95^{\circ} \mathrm{C}$ followed by 39 amplification cycles with $30 \mathrm{~s} /$ $95^{\circ} \mathrm{C}, 45 \mathrm{~s} / 54^{\circ} \mathrm{C}, 45 \mathrm{~s} / 72^{\circ} \mathrm{C}$ and a final elongation $10 \mathrm{~min} / 72^{\circ} \mathrm{C}$ ) was performed with three primers (Figure 1 A, "a": AAGGCGCATAACGATACCAC, "b": TACAATGCAGGCTCCAAACAC, "c": CGAGCACAGGAAGTTCAACA, Eurofins Genomics, Ebersberg/Germany) and the ImmoMix ${ }^{\mathrm{TM}}$ kit (Bioline, Cat. No BIO-25020) according to the manufacturer's instructions.

\subsection{RNA isolation and cDNA synthesis and sequencing}

RNA precipitation was performed with TRIsure ${ }^{\mathrm{TM}}$ (Bioline, London/UK) following to the manufacturer's instructions, from deep-frozen iBAT. Precipitated RNA was loaded to spin columns (SV Total RNA Isolation System, Promega, Cat\# Z3105), centrifuged for 1 min with 12,000 x g and further processed according to the supplier's instructions. RNA concentration was determined spectrophotometrically (Infinite 200 PRO NanoQuant, Tecan). cDNA synthesis was performed with $1 \mu \mathrm{g}$ RNA (SensifAST ${ }^{\mathrm{Tm}}$ cDNA Synthesis Kit, Bioline, Cat\# BIO-65053), according to the manufacturer's instructions. PCR (denaturation: $10 \mathrm{~min} / 95^{\circ} \mathrm{C}$ followed by 30 amplification cycles with $1 \mathrm{~min} / 95^{\circ} \mathrm{C}, 30 \mathrm{~s} / 54{ }^{\circ} \mathrm{C}, 40 \mathrm{~s} / 72^{\circ} \mathrm{C}$ and a final elongation $5 \mathrm{~min} / 72^{\circ} \mathrm{C}$ ) was performed with primers (“d”: cggagtttcagcttgcctggca, "e": tcgcacagcttggtacgcttgg, Eurofins Genomics, Ebersberg/Germany, Figure $1 \mathrm{~A}$ ) and products were separated by gel electrophoresis on a $1 \%$ agarose gel. Separated PCR products were visualized under a UV light, cut, immediately weighed and stored at $-20^{\circ} \mathrm{C}$. PCR products were purified with the Wizard SV Genomic DNA Purification System (Promega, Cat\# A2361), and sent in to a commercial sequencing platform (Eurofins Genomics, Ebersberg/Germany). Analysis of sequencing results was performed with the "Benchling" platform (https://www.benchling.com/). 


\subsection{Protein expression analysis by SDS-Page and Western Blot}

Protein was isolated from interscapular BAT, homogenized in $10 \mu 1 / \mathrm{mg}$ isolation buffer (50 mM Tris, $1 \%$ NP-40, $0.25 \%$ sodium deoxycholate, $150 \mathrm{mM} \mathrm{NaCl}, 1 \mathrm{mM}$ EDTA) containing $0.1 \%$ phosphatase (Sigma-Aldrich, St. Louis MO/USA) and $0.1 \%$ protease inhibitor cocktail (Sigma-Aldrich, St. Louis MO/USA) with a dispersing device (Miccra D-1, Miccra GmbH, Heitersheim/Germany). The homogenized samples were centrifuged $15 \mathrm{~min}$ at $4^{\circ} \mathrm{C}$ with $14.000 \mathrm{rcf}$. The clear layer of the supernatant was isolated by pipetting and centrifuged again. Samples were cleared from residual fat by a second extraction of the clear phase with a syringe. Protein concentrations were determined with the Pierce ${ }^{\mathrm{TM}}$ BCA Protein Assay Kit (ThermoScientific, Rockford IL/USA) according to the manufacturer's instructions. For protein detection, $30 \mu \mathrm{g}$ protein were separated in a $12.5 \%$ SDS-PAGE and transferred to a nitrocellulose membrane. Subsequently, primary antibody was applied to detect UCP1 (ab23841, Abcam, UK, 1:5000) followed by primary antibody detection using an IR-dye conjugated secondary antibody (IRDye 800CW, LI-COR, Lincoln NE/USA, 1:20000). The IR signal was detected with the Azure Sapphire ${ }^{\mathrm{TM}}$ biomolecular imager (azure biosystems, Dublin CA/USA). Image analysis was conducted with the Image Studio ${ }^{\mathrm{TM}}$ Lite software version 5.2.

\subsection{DNA Extraction and 16S rRNA Sequencing}

Cecal contents were collected together with other tissues and immediately snap frozen in liquid nitrogen and stored at $-80^{\circ} \mathrm{C}$. DNA isolation, library preparation and sequencing were performed at the ZIEL Core Facility Microbiome of the Technical University of Munich. Briefly, DNA was extracted using previously published protocols (Klindworth et al. 2013). For the assessment of bacterial communities primers specifically targeting the V3-V4 region of the bacterial 16S rRNA (Forward-Primer (341FCCTACGGGNGGCWGCAG; Reverse-Primer (785r-ovh): GACTACHVGGGTATCTAATCC) gene including a forward and reverse illumina specific overhang and a barcode were used. Sequencing was performed using an Illumina MISeq DNA platform. Obtained multiplexed sequencing files have been analyzed using the IMNGS platform, which is based on the UPARSE approach for sequence quality check, chimera filtering and cluster formation (Edgar 2013; Lagkouvardos et al. 2016). For the analysis standard values for barcode mismatches, trimming, expected errors and abundance cutoff have been 
used and only sequences between 300 and 600 bp were considered for analysis. Downstream analysis of the IMNGS platform output files were performed using the RHEA R pipeline (Lagkouvardos et al. 2017). In brief, obtained abundances have been normalized and quality of obtained sequences was assessed using rarefaction curves (McMurdie and Holmes 2014). Analysis of alpha diversity, beta diversity and group comparisons have been performed using default settings. Exceptions have been applied for group comparisons for zOTUs and taxonomic levels (abundance cutoff 0.5 and exclusion of alpha diversity measures). Graphical output was modified for presentation using inkscape (https://inkscape.org). Assignment of zOTUs to taxons has been performed using the SILVA database (Version 138.1 (Quast et al. 2013)). Assignment of species to specific zOTUs with EZBioCloud (Yoon et al. 2017).

\subsection{Lipid Extraction and Mass Spectrometry Analysis}

Lipid extraction for quantitative analysis using Lipidyzer ${ }^{\mathrm{TM}}$ platform (SCIEX) was done using an adapted Methyl-tert-butyl-ether (MTBE) extraction protocol. Lipidyzer ${ }^{\mathrm{TM}}$ internal standards mixture was prepared according to the manufacturer's instruction but dissolved in MTBE. To each $50 \mu \mathrm{L}$ plasma aliquot, $50 \mu \mathrm{L}$ water; $50 \mu \mathrm{L}$ Internal Standard, $500 \mu \mathrm{L}$ MTBE and $160 \mu \mathrm{L}$ Methanol was added, shortly vortexed and incubated on a mixer for 30 minutes. $200 \mu \mathrm{L}$ of water was added and centrifuged at 16000g. The supernatant was transferred in vials and the residual phase re-extracted using MTBE: Methanol: Water in the ratio 3:1:1. The collected supernatants were evaporated with a vacuum centrifuge and resuspended in $250 \mu \mathrm{L}$ of $10 \mathrm{mM}$ ammonium acetate in Dichloromethane: Methanol $(50: 50(\mathrm{v} / \mathrm{v}))$.

Samples were analyzed using a QTRAP 5500 (AB SCIEX) equipped with Differential Mobility Spectrometer (DMS) interface (Schneider et al. 2010) operating with SelexION technology, coupled to a Shimadzu Nexera X2 liquid chromatography system. The Lipidyzer platform ${ }^{\mathrm{TM}}$ was operated via the software Analyst version 1.6.8 and Lipidomics workflow manager (SCIEX). A detailed description of this shotgun approach has been previously reported (Lintonen et al. 2014). The Lipidyzer ${ }^{\mathrm{TM}}$ Platform was tuned using the SelexION Tuning Kit (SCIEX) according to the manufacturer's recommendations and a system suitability test was performed using the System Suitability Kit (SCIEX) according to the 
manufacturer's instructions. The Lipidyzer ${ }^{\mathrm{TM}}$ Platform uses $10 \mathrm{mM}$ ammonium acetate in Dichloromethane: Methanol (50:50 (v/v)) as running buffer, Dichloromethane: Methanol (50:50 (v/v)) as rinse $0 \& 1$, 2-propanol as rinses $2 \& 3$, and 1-propanol as a DMS modifier. $50 \mu 1$ of samples were injected for each of the two MRM methods: One with a DMS on and one with DMS off. MRM data acquisition, processing, and quantification was performed automatically by the lipidyzer lipidomics workflow manager. Lipid concentrations are given in $\mathrm{nmol} / \mathrm{ml}$.

\subsection{Data analysis and statistics}

General data analysis was performed with R (version 4.0.3) within R-Studio (version 1.3.1093). Unless otherwise indicated data are represented as means \pm sd or with single values for each mouse. Student's t-tests were performed with the package "ggpubr" (version 0.4.0). Anova, and linear model analysis with the package "stats" (version 4.0.3). Trapezoid area under the curves were calculated using the AUC function of the package "DescTools" (version 0.99.38).

Analysis of alpha diversity was performed with Prism 6 (GraphPad Software Inc., La Jolla CA/USA) using non-parametric Mann-Whitney U test. Beta diversity is visualized using non-metric multidimensional scaling based on generalized UniFrac and tested for significance using PERMANOVA. Differences in zOTUs have been determined using Kruskal-Wallis rank sum test with adjustments for multiple testing using the Benjamini \& Hochberg method.

Multi-omics analysis was performed using R (version 4.0.4) and python (version 3.8.5). Multi-omics factor analysis (MOFA) (Argelaguet et al. 2018; 2020) was used for unsupervised data integration of the lipidome and microbiome data. The mofapy2 python package (version 0.5.8) and the MOFA2 R package (version 1.0.1) (for downstream analysis) were used together with custom visualization tools. Data integration analysis for biomarker discovery using latent variable approaches for 'omics studies (DIABLO) (Singh et al. 2019) was used as a supervised analysis framework. DIABLO generalizes (sparse) partial least-squares discriminant analysis (PLS-DA) for the integration of multiple datasets measured on the same samples. For DIABLO analyses the mixOmics R package (version 6.14.0) (Rohart et al. 2017) was used along with custom code for randomized performance estimation. 


\section{Results}

\section{Deletion of UCP1 Exon 2 leads to a loss of protein expression}

The original mouse used to generate the knockout of UCP1 in the mice used in this study was generated in frame of the EUCOMM program via a "knockout first allele" approach (Skarnes et al. 2011; Pettitt et al. 2009). In order to generate WT $\left(U C P 1^{\text {tmlc }}\right)$, the lacZ and the neomycin resistance cassette were removed from the $\mathrm{UCP}^{\text {tmla }}$ allele, by cross breeding with flippase expressing mice (Figure $1 \mathrm{~A} 1$ ), thus generating WT $\left(U C P 1^{t m l c}\right)$ mice. These mice containing only one flippase recognition target (frt) and two lox P sites flanking exon 2 of the UCP1 gene were crossed with mice expressing Cre-recombinase under the control of the rosa26 promotor (Rosa26Cre/+) (Figure 1 A2). This resulted in the generation of $\mathrm{KO}\left(U C P 1^{\text {tmld }}\right)$ mice by constitutive germline deletion of exon 2 in the UCP1 gene (Figure $1 \mathrm{~A} 3$ ). Deletion of exon 2 was first confirmed by PCR on genomic DNA with one forward and two reverse primers binding to distinct sites of the UCP1 gene (Figure 1 A2 \& A3). As predicted, this resulted in a short (263 bp) product for WT mice (Figure $1 \mathrm{~B}$, primers a-b) and a longer (388 bp) product for KO (Figure $1 \mathrm{~B}$, primers a-c), while HET mice showed both products. Of note, the 1255 bp product generated by the primers a and c in WT and HET is not seen, as the elongation period of the PCR protocol is too short to produce a product of this size. To further investigate the consequences of exon 2 deletion, we performed a RT-PCR on RNA isolated from brown adipose tissue of both WT and KO mice. For the primer pair binding in exon 1 (d) and exon 5 (e) of the UCP1 gene (Figure 1 A), KO showed a smaller product size ( $\sim 500 \mathrm{bp})$ compared to WT mice ( $700 \mathrm{bp})$, as predicted by in-silico PCR (KO: 508 bp, WT: 707 bp, https://genome.ucsc.edu/cgi-bin/hgPcr) (Figure $1 \mathrm{C}$ ). Subsequent sequencing of the WT and KO PCR-products revealed that the deletion of exon 2 causes a frame shift, leading to a premature stop codon in exon 3 (Supplementary Figure 1). Consequently, KO mice do not express UCP1 protein, as confirmed by western blot analysis (Figure 1 D, Supplementary Figure 2).

\section{Thermogenic deficiency leads to decreased body weight in young KO mice}

The loss of the major protein responsible for non-shivering thermogenesis resulted in a clear reduction of interscapular skin surface temperature (iSST) in newborn KO compared to WT mice (Figure 2 A\&B). The loss of one functional UCP1 allele (HET) on the other hand had no implication on iSST in newborn 
pups compared to WT mice (Figure $2 \mathrm{~A} \& \mathrm{~B}$ ). The genotype distribution of offspring from HET/HET breeding pairs (generation F2-F3) did not significantly deviate from the mendelian distribution of 1:2:1 (Figure $2 \mathrm{C}$, Table 1). However, KO mice had lower body weight at weaning (at the age of $\sim 3-4$ weeks) compared to HET and WT mice (Figure 2 D), a phenotype that could be confirmed in the conventional UCP1-KO mouse on 129S1/SvImJ- but not on C57Bl/6J-background (Supplementary Figure 3 A\&C). Irrespectively of the knockout model bodyweight of all three genotypes were similar at $\sim 8$ weeks of age (Figure 2 E, Supplementary Figure 3 B\&D).

In summary this suggests a strain dependent effect of UCP1 depletion on early body weight that recovers with age.

\section{UCP1-KO and WT mice have similar susceptibility to DIO at thermoneutrality}

The susceptibility UCP1-KO mice to diet induced obesity (DIO) under thermoneutral conditions is still a matter of debate. We addressed this controversial question using our novel UCP1-KO model by feeding mice at thermoneutrality a control diet (CD) for 4 weeks followed by 8 weeks of high-fat diet (HFD). At the start of the experiment at the age of 8 weeks, mice of both genotypes had similar body weights (data not shown). Cumulative body weight gain increased with time but was similar between both genotypes (Figure $3 \mathrm{~A}$ ) as indicated by linear model analysis during CD (Duration $\mathrm{P}<0.001$, Genotype $\mathrm{P}=0.491$ ) and HFD (Duration $\mathrm{P}<0.001$, Genotype $\mathrm{P}=0.188$ ) feeding. In line, total energy intake between both genotypes was similar during control diet (CD) and high fat diet (HFD) feeding (Figure $3 \mathrm{~B} \& \mathrm{C}$ ).

We determined body composition in terms of lean and fat mass at different time points of the experiment. Both lean mass (Figure 3 D) and fat mass (Figure 3 E) correlated well with body weight during both feeding regimes, with fat mass being the main contributor to the increase in body weight during HFD feeding $\left(\mathrm{R}^{2}>0.9\right)$, in both WT and KO mice (Figure $\left.3 \mathrm{D} \& \mathrm{E}\right)$.

UCP1 knockout mice have been described to be metabolically more efficient (Feldmann et al. 2009; von Essen et al. 2017; Luijten et al. 2019), thus incorporating more fat mass per unit of energy intake. We addressed this question by linear model analysis of cumulative fat mass gain versus cumulative energy 
intake over the experimental period (Figure $3 \mathrm{~F}$ ). There was no difference in the correlation of fat mass gain and energy intake between genotypes, consequently both UCP1-WT and UCP1-KO mice showed similar metabolic efficiency. Of note, this result was confirmed by determining metabolic efficacy as the percentage of food energy stored as fat mass, as described previously (von Essen et al. 2017) (Supplementary Figure 4 A\&B). The similarity in fat mass of both UCP1-WT and UCP1-KO determined by NMR was reinforced by dissected weights of iWAT, eWAT and iBAT (Figure 3 G-I). Collectively these data analyses demonstrate that UCP1 ablation neither affected energy intake, nor body adiposity, nor metabolic efficacy when mice were kept at thermoneutral conditions.

\section{Plasma lipid composition of UCP1-KO and UCP1-WT mice is comparable}

Activated BAT can clear substantial amounts of lipids from circulation (Heine et al. 2018; Bartelt et al. 2011). To study whether UCP1 ablation affected systemic lipid metabolism, a targeted lipidomic approach on plasma samples was performed. Lipid class composition was similar between both genotypes. Only cholesteryl esters (CE) were significantly more abundant in UCP1-WT compared to UCP1-KO mice (Figure 4 A). Concentration of CE, ceramides (CER), hexosylceramides (HCER) and sphingomyelins (SM) were significantly higher in UCP1-WT mice (Figure 4 B). However, fold changes (FC) between to UCP1-KO were rather small $\left(\mathrm{CE}, \mathrm{FC}_{\mathrm{w} t / \mathrm{ko}}=1.13 ; \mathrm{CER}, \mathrm{FC}_{\mathrm{w} t \mathrm{ko}}=1.17 ; \mathrm{HCER}^{\mathrm{F}} \mathrm{C}_{\mathrm{w} t / \mathrm{ko}}=\right.$ 1.16; $\mathrm{SM}, \mathrm{FC}_{\mathrm{w} t \mathrm{ko}}=1.1$ ). The similarity of plasma lipid composition between both genotypes was confirmed by principal component analysis (PCA) of composition (Figure $4 \mathrm{C}$ ) and concentration (Figure 4 D) on a lipid species level.

Collectively, these data demonstrate that ablation of UCP1 did have only minor effects on steady state systemic lipid metabolism at thermoneutrality.

\section{Lack of UCP1 is associated with the abundance of specific microbial genera}

The gut microbiome influences host metabolism (Tremaroli and Bäckhed 2012) and studies demonstrate an effect of microbiome composition on UCP1 expression (Wu et al. 2019) and thermogenesis (Ziętak et al. 2016). Consequently, we investigated whether UCP1 expression alters microbiome composition, by comparing the cecal microbiomes of UCP1-WT and UCP1-KO mice. Similar bacterial richness 
(alpha-diversity) was observed between genotypes by 16S rRNA analysis (Figure 5 A). However, deletion of UCP1 affected cecal microbial composition demonstrated by differences in beta-diversity between genotypes (Figure $5 \mathrm{~B}$ ). Detailed analysis of the microbial composition revealed four zOTU significantly different between UCP1-KO and UCP1-WT based on unadjusted Kruskal-Wallis rank sum test (Figure $5 \mathrm{C}-\mathrm{F}$ ). After adjustment for multiple comparisons, two of these zOTUs demonstrated a trend to higher abundance in UCP1-KO while the other were significantly more abundant in UCP1-WT mice. These zOTUs could be assigned to Parabacteroides goldsteinii (zOTU3 \& zOTU4) and Desuflovibrio fairfieldensis (zOTU17 and zOTU19), respectively. Interestingly, P. goldsteinii has previously been reported to decrease in HFD induced obesity and diabetes and increase UCP1 expression in iBAT and iWAT in C57BL/6J mice (Wu et al. 2019). These data indicate a connection between UCP1 expression and the gut microbiota and confirm P. goldsteinii as a potential species associated with UCP1.

The microbiome can substantially influence host lipid metabolism (Schoeler and Caesar 2019). As we identified small changes in both lipid metabolism and microbiome composition, we investigated potential interactions between microbiome and lipidome. Therefore, we analyzed the combined lipidome and microbiome data set using supervised (DIABLO PLS-DA) and unsupervised (MOFA) approaches. DIABLO PLS-DA revealed two sets of features that discriminated between UCP1-KO and UCP1-WT mice (Figure 6 A). However, quality assessment by repeated analysis of the dataset with randomly assigned groups (1000 iterations) demonstrated similar good discrimination as between UCP1-KO and UCP1-WT mice (Figure 6 A). Consequently, it was not possible to discriminate the observed difference between UCP1-KO and UCP1-WT from random differences between samples. This assumption was confirmed by unsupervised MOFA demonstrating no separation of the two genotypes by the two factor groups explaining the highest proportion of variance between UCP1-KO and UCP1WT mice (Figure $6 \mathrm{~B}$ ).

Consequently, no genotype specific interactions between plasma lipid composition and the microbiome were identified.

\section{UCP1-KO mice have similar energy balance at thermoneutrality}


The effect of UCP1 knockout on energy balance regulation was investigated in detail by indirect calorimetry measurements 3-4 weeks after the start of CD or HFD feeding. We observed a clear diurnal pattern of the respiratory exchange ratio during $\mathrm{CD}$ feeding, being higher during the dark phase compared to the light phase (Figure $7 \mathrm{~A} \& \mathrm{~B}$ ) indicating that mice utilized more carbohydrates during the nocturnal activity phase while relying more on fatty acid metabolism during the daytime resting phase. During HFD feeding the respiratory exchange ratio was generally reduced compared to the CD period, demonstrating a shift in substrate utilization towards fatty acid oxidation based in the high fat content of the diet (Figure $7 \mathrm{C} \& \mathrm{D}$ ). However, no differences in respiratory exchange ratio between KO and WT mice were detected during either feeding period (Figure 7 B\&D).

The activation of BAT thermogenesis by feeding (diet-induced thermogenesis) might contribute to total energy expenditure and thus protect WT mice from diet induced obesity (von Essen et al. 2017). To scrutinize these findings, we investigated whether knockout of UCP1 affected energy expenditure in the new mouse model. As expected, metabolic rate in terms of $\mathrm{O}_{2}$ consumption, $\mathrm{CO}_{2}$ production and heat production were subject to diurnal alterations during CD and HFD feeding, increasing during the nocturnal activity phase, and decreasing during the daytime resting phase (Figure 7 E\&G and Supplementary Figure 5 A-F). However, energy expenditure (area under the heat production curve) during the measurements were similar between WT and KO mice at all times (Figure 7 F\&H and Supplementary Figure 5 E\&F). Consequently, knockout of UCP1 did not affect energy expenditure at thermoneutral conditions.

Subsequent to the energy expenditure measurement during HFD feeding, we investigated basal metabolic rate and noradrenaline (NA) induced heat production in fasted mice. KO and WT mice hat similar basal metabolic rate (Figure 7 I) and increased metabolic rates after NA injection, similar to previous observations (Granneman et al. 2003; Meyer et al. 2010). However, WT showed a remarkably higher response upon NA injection compared to KO (Figure 7 J-L), demonstrating the capacity for UCP1 mediated thermogenesis.

In addition to energy expenditure, we measured energy intake and fecal energy excretion during the calorimetry sessions. Faecal energy content was higher during high fat compared to control diet feeding, 
reflecting the increased energy content of the high-fat diet (not shown). However, there was no difference between genotypes in either feeding period (Figure $8 \mathrm{~A} \& \mathrm{~B}$ ). This was also true for excreted (Figure $8 \mathrm{C} \& \mathrm{D}$ ) and ingested (Figure $8 \mathrm{E} \& \mathrm{~F}$ ) energy during the calorimetry sessions. Consequently, energy balance (ingested energy - excreted energy - AUC of heat production) was unaffected by the deletion of UCP1 under thermoneutral conditions (Figure 8 G\&H). 


\section{Discussion}

We characterize for the first time a novel UCP1 knockout model, as an alternative to the established and widely used UCP1-KO mouse, generated by Leslie Kozak and coworkers (Enerbäck et al. 1997). In regard of the conflicting data published so far on the DIO susceptibility of this established and widely used UCP1-KO model, there is an urgent need for new UCP1-KO models generated by cutting edge transgenic technologies to enable robust validation of metabolic functions for UCP1. Comprehensive metabolic phenotyping of the novel UCP1-KO mouse model presented in this study will help to clarify the role of UCP1 in brown and brite/beige adipose tissues for energy balance regulation, contrasting diet- and cold-induced non-shivering thermogenesis. Down this line, another UCP1-KO mouse model lacking functional UCP1 due to a SNP at nucleotide 38 of exon 5 of the UCP1 gene will also be instrumental for comparative studies (Bond and Ntambi 2018).

The aims of the study were to provide a basal characterization of the constitutive UCP1 knockout mouse and to validate this model by comparison with the results of previous studies on the established UCP1KO mouse, especially in light of the still ongoing debate whether (Feldmann et al. 2009; von Essen et al. 2017; Rowland et al. 2016; Luijten et al. 2019; Pahlavani et al. 2019) or not (Enerbäck et al. 1997; Liu et al. 2003; Zietak and Kozak 2016; Winn et al. 2017; Maurer et al. 2020) the knockout of UCP1 renders mice more susceptible to DIO at thermoneutral conditions.

At standard housing conditions $\left(\sim 23^{\circ} \mathrm{C}\right.$ ambient temperature $)$ mice rely on constantly active thermogenesis to maintain normothermia. Mice lacking UCP1 recruit other thermogenic mechanisms to defend body temperature at these conditions and thus in contrast to WT mice are protected against DIO obesity (Keipert et al. 2020; T. Wang et al. 2008; Liu et al. 2003). Similarly, UCP1-KO pups showed decreased body temperature (iSST) in the present study and decreased weight after weaning, confirming the significance of UCP1 as an efficient mechanism to defend body temperature in early life. Interestingly, differences in body weight after weaning could be seen in pups of the conventional UCP1KO mouse on a 129S1/SvImJ but not on a C57BL/6J background. Indeed, the effect of UCP1 knockout depends on the genetic background since congenic 129S1/SvImJ or C57BL/6J UCP1 knockout mice are cold sensitive, while their F1-hybrids are not (Hofmann et al. 2001). 
Once the need for thermoregulatory heat production is eliminated by housing mice in their thermoneutral zone $\left(27-30^{\circ} \mathrm{C}\right)$ the effect of UCP1 deletion becomes inconclusive. Results from several studies suggest that at thermoneutrality UCP1-KO mice are more susceptible to DIO due to the lack of diet-induced thermogenesis (von Essen et al. 2017; Feldmann et al. 2009; Rowland et al. 2016; Luijten et al. 2019), a mechanism activating BAT thermogenesis enabling rodents to increase their energy expenditure to avoid excessive weight gain caused by overfeeding (Bachman et al. 2002; Rothwell and Stock 1979). Thus, it seems plausible that mice lacking UCP1 are more susceptible to DIO at thermoneutrality (Feldmann et al. 2009; Luijten et al. 2019; von Essen et al. 2017; Rowland et al. 2016) considering UCP1 as the main contributor to BAT thermogenesis. We investigated this phenomenon by comprehensive metabolic analysis of our novel UCP1-KO model. Total energy expenditure was similar in both $\mathrm{KO}$ and WT mice and did not differ during the nocturnal feeding period, indicating no effect of UCP1 ablation on diet induced thermogenesis. Further, considering the similarities in body weight gain, food intake and metabolic efficiency between $\mathrm{KO}$ and WT animals there is no evidence for a more DIO susceptible phenotype of UCP1-KO mice. These findings are in line with various studies on the established UCP1-KO mouse (Enerbäck et al. 1997; Zietak and Kozak 2016; Winn et al. 2017; Maurer et al. 2020) and a second recently described UCP1-KO mouse (Bond and Ntambi 2018). Based on the combined evidence of different UCP1-KO models we conclude that energy balance regulation and the development of DIO are not affected by the presence of UCP1 at thermoneutrality.

For future studies, a major advantage and novelty of our mouse model compared to other available UCP1-KO models (Enerbäck et al. 1997; Bond and Ntambi 2018) is the option to induce conditional Cre-mediated deletion of Exon 2 in the UCP1 gene, using tamoxifen- or digitonin-inducible Cresystems. Although, we described the constitutive UCP1-KO, this system enables conditional cell-type specific or age-dependent knock-out of UCP1. This is of significance to investigate the role of alternative mechanisms for non-shivering thermogenesis that might be recruited due to the lack of UCP1 in early life stages. So far, the only available inducible model was the UCP1-DTR mouse, expressing the diphtheria toxin receptor (DTR) under control of the UCP1 promoter, thus depleting UCP1 expressing cells (Rosenwald et al. 2013; Challa et al. 2020). This provided first insights about the contribution of brite adipocytes to energy expenditure (Challa et al. 2020). In contrast, our model will allow the selective 
ablation of UCP1 in distinct cell types while leaving these cells otherwise functional. Further research on inducible UCP1-KO mice based on our knockout strategy will help to study the recruitment of alternative thermogenic mechanism and to clarify the role of individual thermogenic adipocytes to nonshivering thermogenesis.

In summary, we provide evidence that the abundance of UCP1 does not influence energy metabolism at thermoneutrality and provide a new mouse model as foundation for a better understanding of the contribution of UCP1 in different cell types or life stages to energy metabolism. 


\section{Acknowledgments}

We thank the animal caretakers for their support with animal work. We thank Katherina Schnabl for excellent technical support with the indirect calorimetry as well as Johanna Bruder and Josef Oeckl for assisting during tissue sampling.

This work was financed by the "Nutribrite" grant (Deutsche Forschungsgemeinschaft (DFG) \#KL973/13-1 \& French Agence Nationale de la Recherche \#ANR-15-CE14-0033), the Collaborative Research Center (DFG-CRC1371:P13), and the Else Kröner Fresenius Foundation (EKFS) to M.K.; as well as a European Commission grant EUCOMM (EU-FP6, LSHM-CT-2005-01893). Contributions by N.K. and J.K.P. are funded by the Bavarian State Ministry of Science and the Arts within the framework of the Bavarian Research Institute for Digital Transformation (bidt). J.H. was supported by a DFG grant with the project-ID: 335447727 - SFB 1328.

\section{Author contributions}

S.D. conceived the study design, planned, and performed the mouse experiments, conducted molecular analyses, analyzed, and interpreted data, and wrote the manuscript.

A.S. assisted tissue sampling, analyzed, and interpreted microbiome data, and revised the manuscript.

M.W. conceived the study design, established the animal breeding.

S.M. contributed to data collection and revised the manuscript.

W.W. generated and provided the founder mice and revised the manuscript.

S.M. generated the founder mice and revised the manuscript.

M.H.A. generated the founder mice and revised the manuscript.

R.K. generated the founder mice and revised the manuscript.

A.W. performed the lipidomic analysis.

M.F. performed the lipidomic analysis and revised the manuscript.

J.H. performed the lipidomic analysis and revised the manuscript.

N.K. performed multi-omics analysis and revised the manuscript.

J.P. performed multi-omics analysis and revised the manuscript.

M.K. conceived the study design, contributed to the interpretation of the data, edited the manuscript, 
and acquired funding.

All authors approved the final version of the manuscript.

\section{Conflict of Interest}

The authors declare that the research was conducted in the absence of any commercial or financial relationships that could be construed as a potential conflict of interest. 


\section{References}

Argelaguet, Ricard, Damien Arnol, Danila Bredikhin, Yonatan Deloro, Britta Velten, John C. Marioni, and Oliver Stegle. 2020. "MOFA+: A Statistical Framework for Comprehensive Integration of MultiModal Single-Cell Data.” Genome Biology 21 (1): 111. https://doi.org/10.1186/s13059-020-02015-1.

Argelaguet, Ricard, Britta Velten, Damien Arnol, Sascha Dietrich, Thorsten Zenz, John C Marioni, Florian Buettner, Wolfgang Huber, and Oliver Stegle. 2018. "Multi-Omics Factor Analysis-a Framework for Unsupervised Integration of Multi-omics Data Sets.” Molecular Systems Biology 14 (6). https://doi.org/10.15252/msb.20178124.

Bachman, Eric S., Harveen Dhillon, Chen Yu Zhang, Saverio Cinti, Antonio C. Bianco, Brian K. Kobilka, and Bradford B. Lowell. 2002. "BAR Signaling Required for Diet-Induced Thermogenesis and Obesity Resistance.” Science 297 (5582): 843-45. https://doi.org/10.1126/science.1073160.

Bartelt, Alexander, Oliver T. Bruns, Rudolph Reimer, Heinz Hohenberg, Harald Ittrich, Kersten Peldschus, Michael G. Kaul, et al. 2011. "Brown Adipose Tissue Activity Controls Triglyceride Clearance.” Nature Medicine 17 (2): 200-206. https://doi.org/10.1038/nm.2297.

Becher, Tobias, Srikanth Palanisamy, Daniel J. Kramer, Mahmoud Eljalby, Sarah J. Marx, Andreas G. Wibmer, Scott D. Butler, et al. 2021. "Brown Adipose Tissue Is Associated with Cardiometabolic Health." Nature Medicine 27 (1): 58-65. https://doi.org/10.1038/s41591-020-1126-7.

Bond, Laura M., and James M. Ntambi. 2018. "UCP1 Deficiency Increases Adipose Tissue Monounsaturated Fatty Acid Synthesis and Trafficking to the Liver." Journal of Lipid Research 59 (2): 224-36. https://doi.org/10.1194/jlr.M078469.

Challa, Tenagne D., Dianne H. Dapito, Elisabeth Kulenkampff, Elke Kiehlmann, Caroline Moser, Leon Straub, Wenfei Sun, and Christian Wolfrum. 2020. "A Genetic Model to Study the Contribution of Brown and Brite Adipocytes to Metabolism." Cell Reports 30 (10): 3424-3433.e4. https://doi.org/10.1016/j.celrep.2020.02.055. 
Chen, Hui-Feng, Chen-Ming Hsu, and Yi-Shuian Huang. 2018. “ CPEB 2-dependent Translation of Long 3'- UTR Ucp1 MRNA Promotes Thermogenesis in Brown Adipose Tissue .” The EMBO Journal 37 (20). https://doi.org/10.15252/embj.201899071.

Edgar, Robert C. 2013. "UPARSE: Highly Accurate OTU Sequences from Microbial Amplicon Reads.” Nature Methods 10 (10): 996-98. https://doi.org/10.1038/nmeth.2604.

Enerbäck, Sven, Anders Jacobsson, Elizabeth M. Simpson, Carmen Guerra, Hitoshi Yamashita, Mary Ellen Harper, and Leslie P. Kozak. 1997. “Mice Lacking Mitochondrial Uncoupling Protein Are ColdSensitive but Not Obese.” Nature 387 (6628): 90-94. https://doi.org/10.1038/387090a0.

Essen, Gabriella von, Erik Lindsund, Barbara Cannon, and Jan Nedergaard. 2017. “Adaptive Facultative Diet-Induced Thermogenesis in Wild-Type but Not in UCP1-Ablated Mice." American Journal of Physiology - Endocrinology and Metabolism $313 \quad$ (5): E515-27. https://doi.org/10.1152/ajpendo.00097.2017.

Feldmann, Helena M., Valeria Golozoubova, Barbara Cannon, and Jan Nedergaard. 2009. "UCP1 Ablation Induces Obesity and Abolishes Diet-Induced Thermogenesis in Mice Exempt from Thermal Stress by Living at Thermoneutrality." Cell Metabolism 9 (2): 203-9. https://doi.org/10.1016/j.cmet.2008.12.014.

Fischer, Alexander W., Janina Behrens, Frederike Sass, Christian Schlein, Markus Heine, Paul Pertzborn, Ludger Scheja, and Joerg Heeren. 2020. "Brown Adipose Tissue Lipoprotein and Glucose Disposal Is Not Determined by Thermogenesis in Uncoupling Protein 1-Deficient Mice.” Journal of Lipid Research 61 (11): 1377-89. https://doi.org/10.1194/jlr.RA119000455.

Fischer, Alexander W., Barbara Cannon, and Jan Nedergaard. 2018. “Optimal Housing Temperatures for Mice to Mimic the Thermal Environment of Humans: An Experimental Study." Molecular Metabolism 7 (January): 161-70. https://doi.org/10.1016/j.molmet.2017.10.009.

Fromme, Tobias, Kristina Hüttinger, Stefanie Maurer, Yongguo Li, Thomas Gantert, Jarlei Fiamoncini, Hannelore Daniel, Sören Westphal, and Martin Klingenspor. 2019. "Bile Acid Supplementation 
Decreases Body Mass Gain in C57BL/6J but Not 129S6/SvEvTac Mice without Increasing Energy Expenditure." Scientific Reports 9 (1): 131. https://doi.org/10.1038/s41598-018-37464-z.

Glick, Z., R. J. Teague, and G. A. Bray. 1981. “Brown Adipose Tissue: Thermic Response Increased by a Single Low Protein, High Carbohydrate Meal.” Science 213 (4512): 1125-27. https://doi.org/10.1126/science.7268419.

Gnad, Thorsten, Saskia Scheibler, Ivar Von Kugelgen, Camilla Scheele, Ana Kilic, Anja Glode, Linda S. Hoffmann, et al. 2014. “Adenosine Activates Brown Adipose Tissue and Recruits Beige Adipocytes via A2A Receptors.” Nature 516 (7531): 395-99. https://doi.org/10.1038/nature13816.

Granneman, J. G., M. Burnazi, Z. Zhu, and L. A. Schwamb. 2003. "White Adipose Tissue Contributes to UCP1-Independent Thermogenesis." American Journal of Physiology - Endocrinology and Metabolism 285 (6 48-6): E1230-36. https://doi.org/10.1152/ajpendo.00197.2003.

Heine, Markus, Alexander W. Fischer, Christian Schlein, Caroline Jung, Leon G. Straub, Kristina Gottschling, Nils Mangels, et al. 2018. "Lipolysis Triggers a Systemic Insulin Response Essential for Efficient Energy Replenishment of Activated Brown Adipose Tissue in Mice.” Cell Metabolism 28 (4): 644-655.e4. https://doi.org/10.1016/j.cmet.2018.06.020.

Heldmaier, Gerhard. 1975. "Metabolic and Thermoregulatory Responses to Heat and Cold in the Djungarian Hamster, Phodopus Sungorus.” Journal of Comparative Physiology a B 102 (2): 115-22. https://doi.org/10.1007/BF00691297.

Hofmann, Wolfgang E., Xiaotuan Liu, Christie M. Bearden, Mary Ellen Harper, and Leslie P. Kozak. 2001. "Effects of Genetic Background on Thermoregulation and Fatty Acid-Induced Uncoupling of Mitochondria in UCP1-Deficient Mice.” Journal of Biological Chemistry 276 (15): 12460-65. https://doi.org/10.1074/jbc.M100466200.

Kajimura, Shingo, Bruce M. Spiegelman, and Patrick Seale. 2015. "Brown and Beige Fat: Physiological Roles beyond Heat Generation.” Cell Metabolism. Cell Press. https://doi.org/10.1016/j.cmet.2015.09.007. 
Keipert, Susanne, Dominik Lutter, Bjoern O. Schroeder, Daniel Brandt, Marcus Ståhlman, Thomas Schwarzmayr, Elisabeth Graf, et al. 2020. "Endogenous FGF21-Signaling Controls Paradoxical Obesity Resistance of UCP1-Deficient Mice.” Nature Communications $11 \quad$ (1): 624. https://doi.org/10.1038/s41467-019-14069-2.

Klindworth, Anna, Elmar Pruesse, Timmy Schweer, Jörg Peplies, Christian Quast, Matthias Horn, and Frank Oliver Glöckner. 2013. "Evaluation of General 16S Ribosomal RNA Gene PCR Primers for Classical and Next-Generation Sequencing-Based Diversity Studies.” Nucleic Acids Research 41 (1). https://doi.org/10.1093/nar/gks808.

Lagkouvardos, Ilias, Sandra Fischer, Neeraj Kumar, and Thomas Clavel. 2017. "Rhea: A Transparent and Modular R Pipeline for Microbial Profiling Based on 16S RRNA Gene Amplicons.” PeerJ 2017 (1): e2836. https://doi.org/10.7717/peerj.2836.

Lagkouvardos, Ilias, Divya Joseph, Martin Kapfhammer, Sabahattin Giritli, Matthias Horn, Dirk Haller, and Thomas Clavel. 2016. "IMNGS: A Comprehensive Open Resource of Processed 16S RRNA Microbial Profiles for Ecology and Diversity Studies." Scientific Reports 6 (1): 1-9. https://doi.org/10.1038/srep33721.

Li, Yongguo, Katharina Schnabl, Sarah Madeleine Gabler, Monja Willershäuser, Josefine Reber, Angelos Karlas, Sanna Laurila, et al. 2018. "Secretin-Activated Brown Fat Mediates Prandial Thermogenesis to Induce Satiation.” Cell $175 \quad$ (6): 1561-1574.e12. https://doi.org/10.1016/j.cell.2018.10.016.

Lintonen, Tuulia P.I., Paul R.S. Baker, Matti Suoniemi, Baljit K. Ubhi, Kaisa M. Koistinen, Eva Duchoslav, J. Larry Campbell, and Kim Ekroos. 2014. "Differential Mobility Spectrometry-Driven Shotgun Lipidomics.” Analytical Chemistry. https://doi.org/10.1021/ac5021744.

Liu, Xiaotuan, Martin Rossmeisl, Jennifer McClaine, and Leslie P. Kozak. 2003. "Paradoxical Resistance to Diet-Induced Obesity in UCP1-Deficient Mice.” Journal of Clinical Investigation 111 (3): 399-407. https://doi.org/10.1172/jci15737. 
Lowell, Bradford B., Vedrana S-Susulic, Andreas Hamann, Joel A. Lawitts, Jean Himms-Hagen, Bert B. Boyer, Leslie P. Kozak, and Jeffrey S. Flier. 1993. "Development of Obesity in Transgenic Mice after Genetic Ablation of Brown Adipose Tissue." Nature 366 (6457): 740-42. https://doi.org/10.1038/366740a0.

Luijten, Ineke H.N., Helena M. Feldmann, Gabriella von Essen, Barbara Cannon, and Jan Nedergaard. 2019. "In the Absence of UCP1-Mediated Diet-Induced Thermogenesis, Obesity Is Augmented Even in the Obesity-Resistant 129S Mouse Strain.” American Journal of Physiology - Endocrinology and Metabolism 316 (5): E729-40. https://doi.org/10.1152/ajpendo.00020.2019.

Maurer, Stefanie F., Tobias Fromme, Lawrence I. Grossman, Maik Hüttemann, and Martin Klingenspor. 2015. "The Brown and Brite Adipocyte Marker Cox7a1 Is Not Required for Non-Shivering Thermogenesis in Mice." Scientific Reports 5 (1): 17704. https://doi.org/10.1038/srep17704.

Maurer, Stefanie F., Tobias Fromme, Sabine Mocek, Anika Zimmermann, and Martin Klingenspor. 2020. "Uncoupling Protein 1 and the Capacity for Nonshivering Thermogenesis Are Components of the Glucose Homeostatic System.” American Journal of Physiology - Endocrinology and Metabolism 318 (2): E198-215. https://doi.org/10.1152/ajpendo.00121.2019.

McMurdie, Paul J., and Susan Holmes. 2014. "Waste Not, Want Not: Why Rarefying Microbiome Data Is Inadmissible.” Edited by Alice Carolyn McHardy. PLoS Computational Biology 10 (4): e1003531. https://doi.org/10.1371/journal.pcbi.1003531.

Meyer, Carola W., Monja Willershäuser, Martin Jastroch, Bryan C. Rourke, Tobias Fromme, Rebecca Oelkrug, Gerhard Heldmaier, and Martin Klingenspor. 2010. "Adaptive Thermogenesis and Thermal Conductance in Wild-Type and UCP1-KO Mice." American Journal of Physiology - Regulatory $\begin{array}{lllll}\text { Integrative and } & \text { Comparative Physiology } 299 & \text { (5): R1396-1406. }\end{array}$ https://doi.org/10.1152/ajpregu.00021.2009.

Pahlavani, Mandana, Latha Ramalingam, Emily K. Miller, Shane Scoggin, Kalhara R. Menikdiwela, Nishan S. Kalupahana, William T. Festuccia, and Naima Moustaid-Moussa. 2019. "Eicosapentaenoic Acid Reduces Adiposity, Glucose Intolerance and Increases Oxygen Consumption Independently of 
Uncoupling Protein 1.” Molecular Nutrition and Food Research 63 (7): 1800821. https://doi.org/10.1002/mnfr.201800821.

Pettitt, Stephen J., Qi Liang, Xin Y. Rairdan, Jennifer L. Moran, Haydn M. Prosser, David R. Beier, Kent C. Lloyd, Allan Bradley, and William C. Skarnes. 2009. “Agouti C57BL/6N Embryonic Stem Cells for Mouse Genetic Resources." Nature Methods 6 (7): 493-95. https://doi.org/10.1038/nmeth.1342.

Quast, Christian, Elmar Pruesse, Pelin Yilmaz, Jan Gerken, Timmy Schweer, Pablo Yarza, Jörg Peplies, and Frank Oliver Glöckner. 2013. “The SILVA Ribosomal RNA Gene Database Project: Improved Data Processing and Web-Based Tools." Nucleic Acids Research $41 \quad$ (D1). https://doi.org/10.1093/nar/gks1219.

Rohart, Florian, Benoît Gautier, Amrit Singh, and Kim-Anh Lê Cao. 2017. "MixOmics: An R Package for 'omics Feature Selection and Multiple Data Integration." Edited by Dina Schneidman. PLOS Computational Biology 13 (11): e1005752. https://doi.org/10.1371/journal.pcbi.1005752.

Rosenwald, Matthias, Aliki Perdikari, Thomas Rülicke, and Christian Wolfrum. 2013. "Bi-Directional Interconversion of Brite and White Adipocytes." Nature Cell Biology 15 (6): 659-67. https://doi.org/10.1038/ncb2740.

Rothwell, Nancy J., and Michael J. Stock. 1979. “A Role for Brown Adipose Tissue in Diet-Induced Thermogenesis.” Nature 281 (5726): 31-35. https://doi.org/10.1038/281031a0.

Rowland, Leslie A., Santosh K. Maurya, Naresh C. Bal, Leslie Kozak, and Muthu Periasamy. 2016. "Sarcolipin and Uncoupling Protein 1 Play Distinct Roles in Diet-Induced Thermogenesis and Do Not Compensate for One Another." Obesity 24 (7): 1430-33. https://doi.org/10.1002/oby.21542.

Schnabl, Katharina, Yongguo Li, and Martin Klingenspor. 2020. "The Gut Hormone Secretin Triggers a Gut-Brown Fat-Brain Axis in the Control of Food Intake.” Experimental Physiology 105 (8): 120613. https://doi.org/10.1113/EP087878.

Schneider, Bradley B., Thomas R. Covey, Stephen L. Coy, Evgeny V. Krylov, and Erkinjon G. Nazarov. 2010. "Planar Differential Mobility Spectrometer as a Pre-Filter for Atmospheric Pressure Ionization 
Mass Spectrometry." International Journal of Mass Spectrometry. https://doi.org/10.1016/j.ijms.2010.01.006.

Schoeler, Marc, and Robert Caesar. 2019. "Dietary Lipids, Gut Microbiota and Lipid Metabolism.” Reviews in Endocrine and Metabolic Disorders. Springer. https://doi.org/10.1007/s11154-019-095120 .

Singh, Amrit, Casey P. Shannon, Benoît Gautier, Florian Rohart, Michaël Vacher, Scott J. Tebbutt, and Kim-Anh Lê Cao. 2019. "DIABLO: An Integrative Approach for Identifying Key Molecular Drivers from Multi-Omics Assays." Edited by Inanc Birol. Bioinformatics 35 (17): 3055-62. https://doi.org/10.1093/bioinformatics/bty1054.

Skarnes, William C., Barry Rosen, Anthony P. West, Manousos Koutsourakis, Wendy Bushell, Vivek Iyer, Alejandro O. Mujica, et al. 2011. “A Conditional Knockout Resource for the Genome-Wide Study of Mouse Gene Function.” Nature 474 (7351): 337-44. https://doi.org/10.1038/nature10163.

Tremaroli, Valentina, and Fredrik Bäckhed. 2012. "Functional Interactions between the Gut Microbiota and Host Metabolism.” Nature. Nature Publishing Group. https://doi.org/10.1038/nature11552.

Wang, Hui, Monja Willershäuser, Angelos Karlas, Dimitris Gorpas, Josefine Reber, Vasilis Ntziachristos, Stefanie Maurer, Tobias Fromme, Yongguo Li, and Martin Klingenspor. 2019. “A Dual Ucp1 Reporter Mouse Model for Imaging and Quantitation of Brown and Brite Fat Recruitment." Molecular Metabolism 20 (February): 14-27. https://doi.org/10.1016/j.molmet.2018.11.009.

Wang, Hui, Monja Willershäuser, Yongguo Li, Tobias Fromme, Katharina Schnabl, Andrea BastHabersbrunner, Samira Ramisch, Sabine Mocek, and Martin Klingenspor. 2021. "Uncoupling Protein1 Expression Does Not Protect Mice from Diet-Induced Obesity." American Journal of PhysiologyEndocrinology and Metabolism 320 (2): E333-45. https://doi.org/10.1152/ajpendo.00285.2020.

Wang, Ting, Youxue Wang, Yasuhide Kontani, Yoshinori Kobayashi, Yuzo Sato, Nozomu Mori, and Hitoshi Yamashita. 2008. "Evodiamine Improves Diet-Induced Obesity in a Uncoupling Protein-1Independent Manner: Involvement of Antiadipogenic Mechanism and Extracellularly Regulated 
Kinase/Mitogen-Activated Protein Kinase Signaling.” Endocrinology 149 (1): 358-66. https://doi.org/10.1210/en.2007-0467.

Winn, Nathan C., Victoria J. Vieira-Potter, Michelle L. Gastecki, Rebecca J. Welly, Rebecca J. Scroggins, Terese M. Zidon, T’Keaya L. Gaines, et al. 2017. "Loss of UCP1 Exacerbates Western DietInduced Glycemic Dysregulation Independent of Changes in Body Weight in Female Mice.” American Journal of Physiology - Regulatory Integrative and Comparative Physiology 312 (1): R74-84. https://doi.org/10.1152/ajpregu.00425.2016.

Wu, Tsung-Ru, Chuan-Sheng Lin, Chih-Jung Chang, Tzu-Lung Lin, Jan Martel, Yun-Fei Ko, David M. Ojcius, Chia-Chen Lu, John D. Young, and Hsin-Chih Lai. 2019. "Gut Commensal Parabacteroides Goldsteinii Plays a Predominant Role in the Anti-Obesity Effects of Polysaccharides Isolated from Hirsutella Sinensis." Gut 68 (2): 248-62. https://doi.org/10.1136/gutjnl-2017-315458.

Yoon, Seok Hwan, Sung Min Ha, Soonjae Kwon, Jeongmin Lim, Yeseul Kim, Hyungseok Seo, and Jongsik Chun. 2017. "Introducing EzBioCloud: A Taxonomically United Database of 16S RRNA Gene Sequences and Whole-Genome Assemblies." International Journal of Systematic and Evolutionary Microbiology. https://doi.org/10.1099/ijsem.0.001755.

Ziętak, Marika, Petia Kovatcheva-Datchary, Lidia H. Markiewicz, Marcus Ståhlman, Leslie P. Kozak, and Fredrik Bäckhed. 2016. “Altered Microbiota Contributes to Reduced Diet-Induced Obesity upon Cold Exposure.” Cell Metabolism 23 (6): 1216-23. https://doi.org/10.1016/j.cmet.2016.05.001.

Zietak, Marika, and Leslie P. Kozak. 2016. "Bile Acids Induce Uncoupling Protein 1-Dependent Thermogenesis and Stimulate Energy Expenditure at Thermoneutrality in Mice." American Journal of Physiology - Endocrinology and Metabolism $310 \quad$ (5): E346-54. https://doi.org/10.1152/ajpendo.00485.2015. 


\section{Figures}

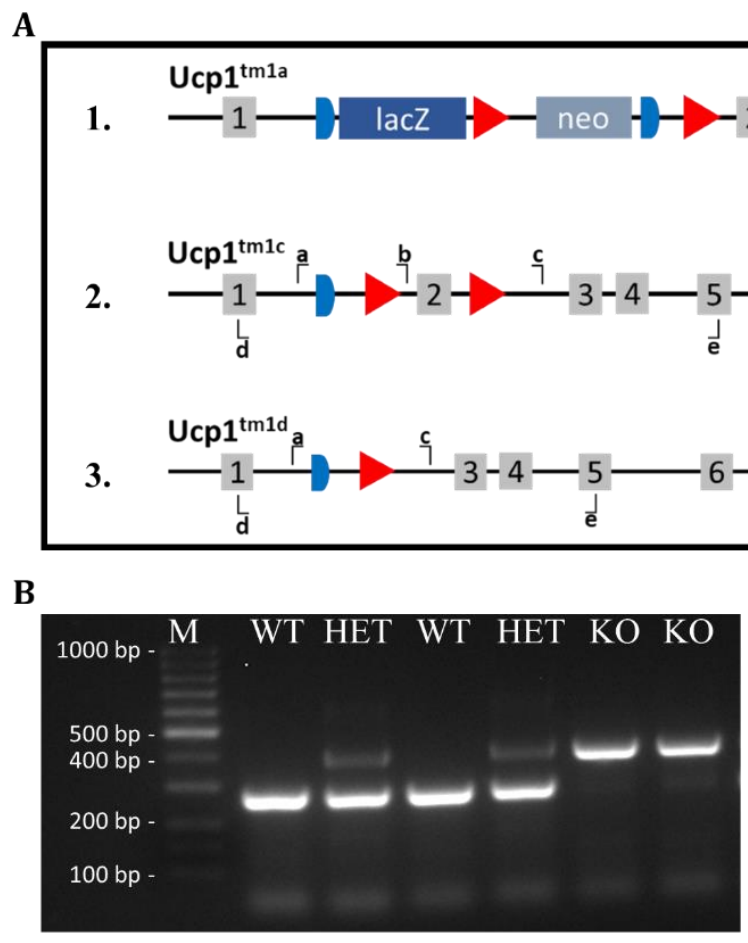

\section{C}

D

$\sim 34 \mathrm{kDa}-$

$\mathrm{KO}$

$\mathrm{M}$

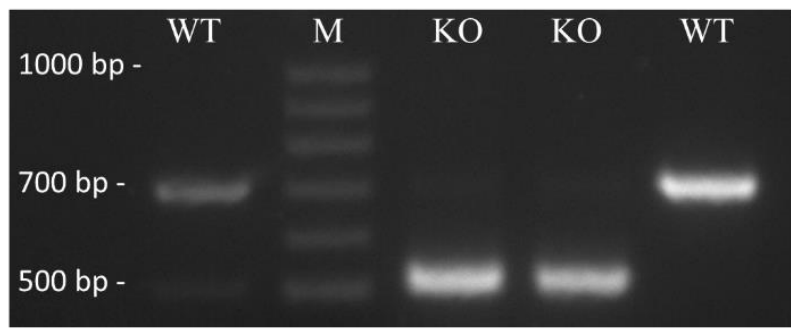

$\sim 25 \mathrm{kDa}-$

\section{Figure 1: Overview and validation of the Ucp1 knockout strategy.}

(A) Breeding scheme for the generation of the Ucp1 knockout. (A1) Ucp1tm1a mice containing three loxP sites (red), two frt sites (blue) as well as a lacZ and a neo cassette were crossed with mice expressing flippase (Flp+). (A2) The resulting Ucp1tm1c (WT) mouse is crossed with a Rosa26CRE/+ mouse, deleting exon 2 of the ucp 1 gene, generating Ucp1tm1d (KO) mice. (A3) Ucp 1tm1c and Ucp1tm1d mice are crossed to generate HET mice. Lower case letters indicating binding positions of primers used for PCR (a-c) and RT-PCR (d\&e). (B) PCR of gDNA from tissue samples of WT, HET and KO mice. (C) RT-PCR products from iBAT of WT and KO mice. (D) Representative western blot analysis for Ucp1 ( $33 \mathrm{kDA})$ in KO, HET and WT mice. See Supplementary Figure 2 for the uncropped image. 
A

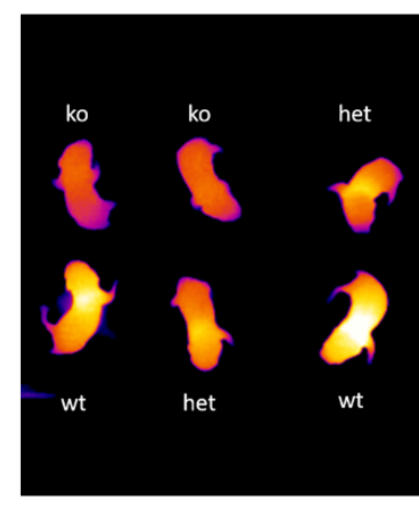

C

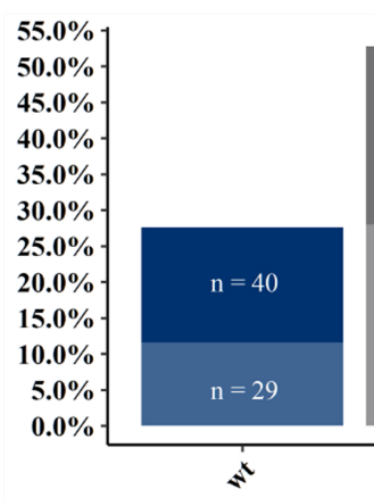

D

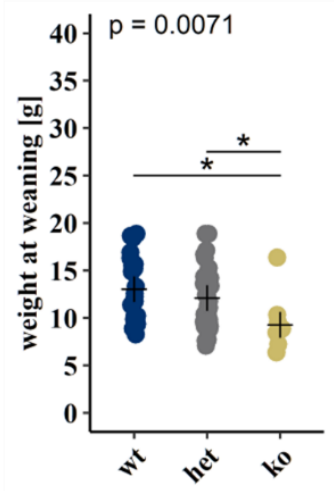

B
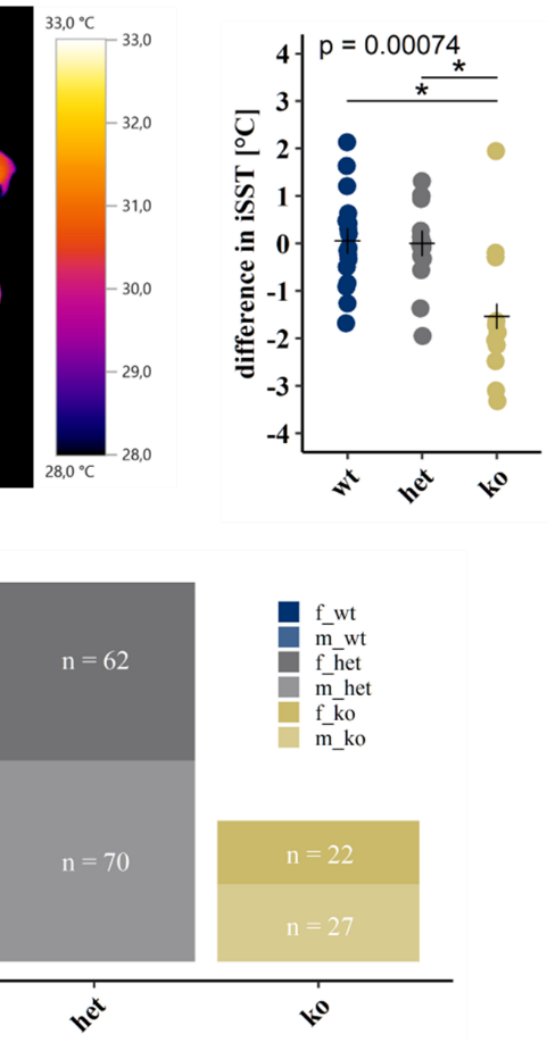

$\mathbf{E}$

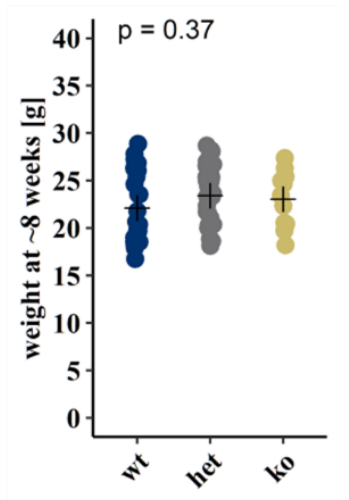

Figure 2: Lack of Ucp1 leads to phenotypic alterations in young mice.

(A) Representative thermal image of newborn pups (2-3 days) of a Ucp1-HET breeding pair. (B) Analysis of interscapular skin surface temperature (iSST, $\mathrm{n}(\mathrm{wt})=17, \mathrm{n}(\mathrm{het})=17, \mathrm{n}(\mathrm{ko})=11, \mathrm{~N}=45$ of 5 litters). (C) Offspring genotype distribution of Ucp1-HET breeding pairs $(\mathrm{n}=15)$ at $23{ }^{\circ} \mathrm{C}$ ambient temperature ( $\mathrm{N}=250)$. (D-E) Body weight of female and male Ucp1-WT ( $\mathrm{n}=23)$, Ucp1-HET $(\mathrm{n}=33)$ and Ucp1-KO (n = 11) mice (D) at weaning and $(\mathbf{E})$ at the age of 8-weeks ( $=67$ of 9 litters). (B,D,E) Crosses indicating group means. 1-Way ANOVA and t-test with bonferroni adjusted p-value, $*=\mathrm{p}$ value $<0.05$. 
A

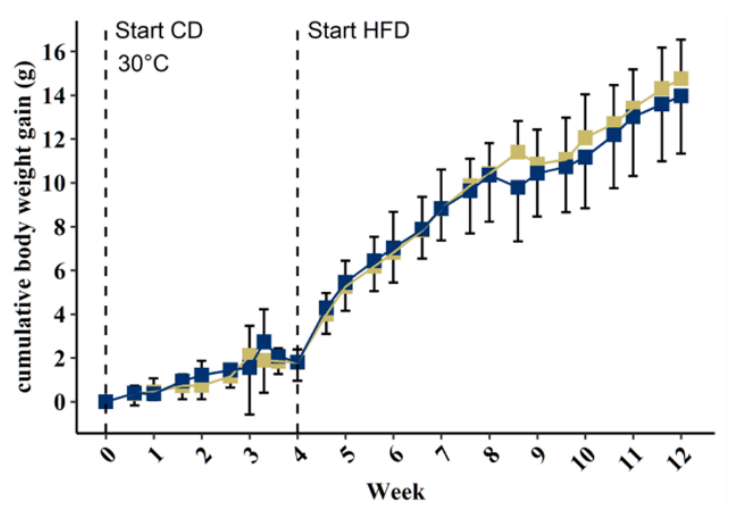

D

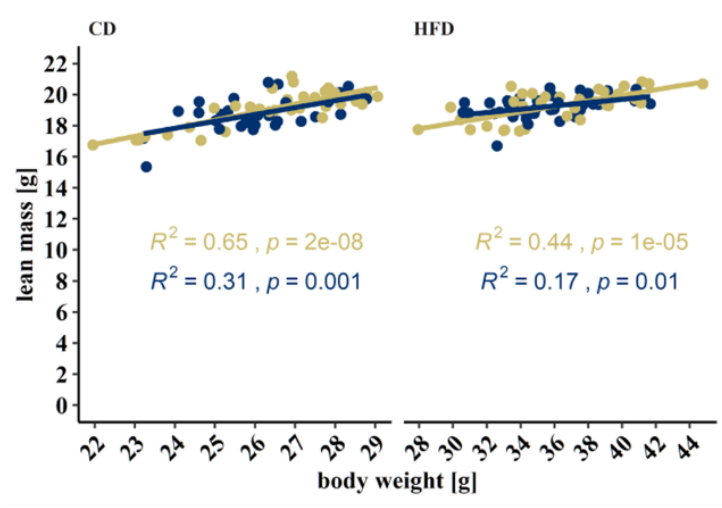

F

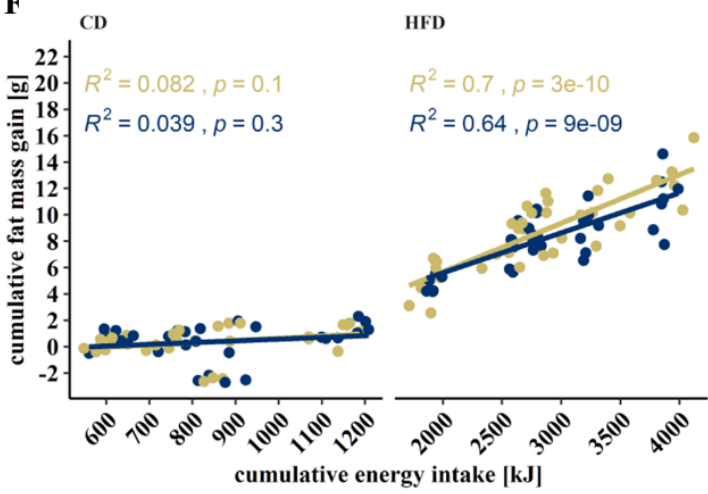

B

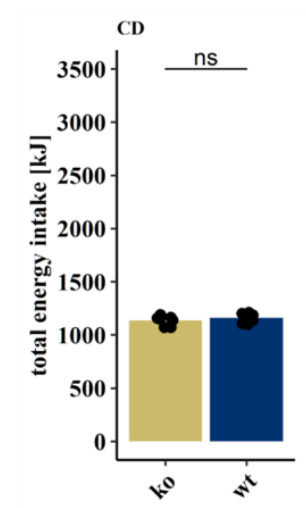

$\mathbf{E}$

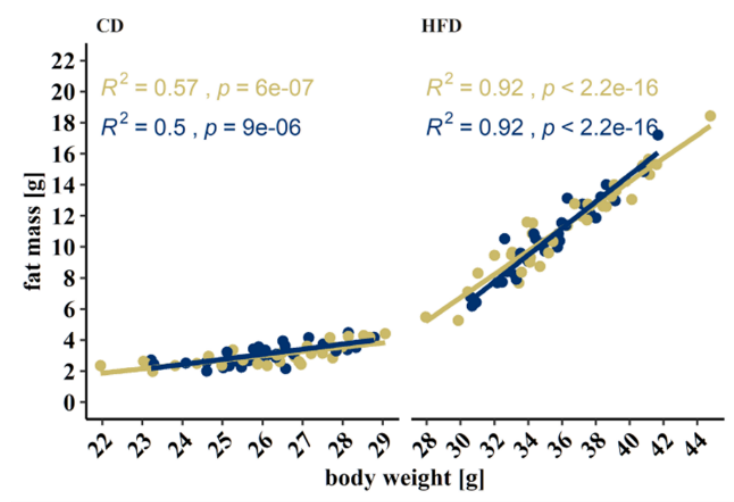

G

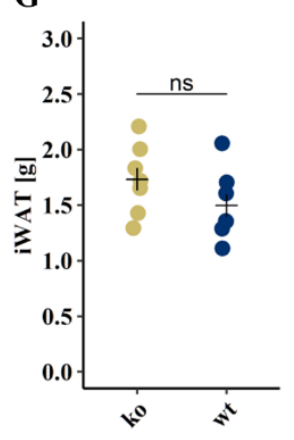

C
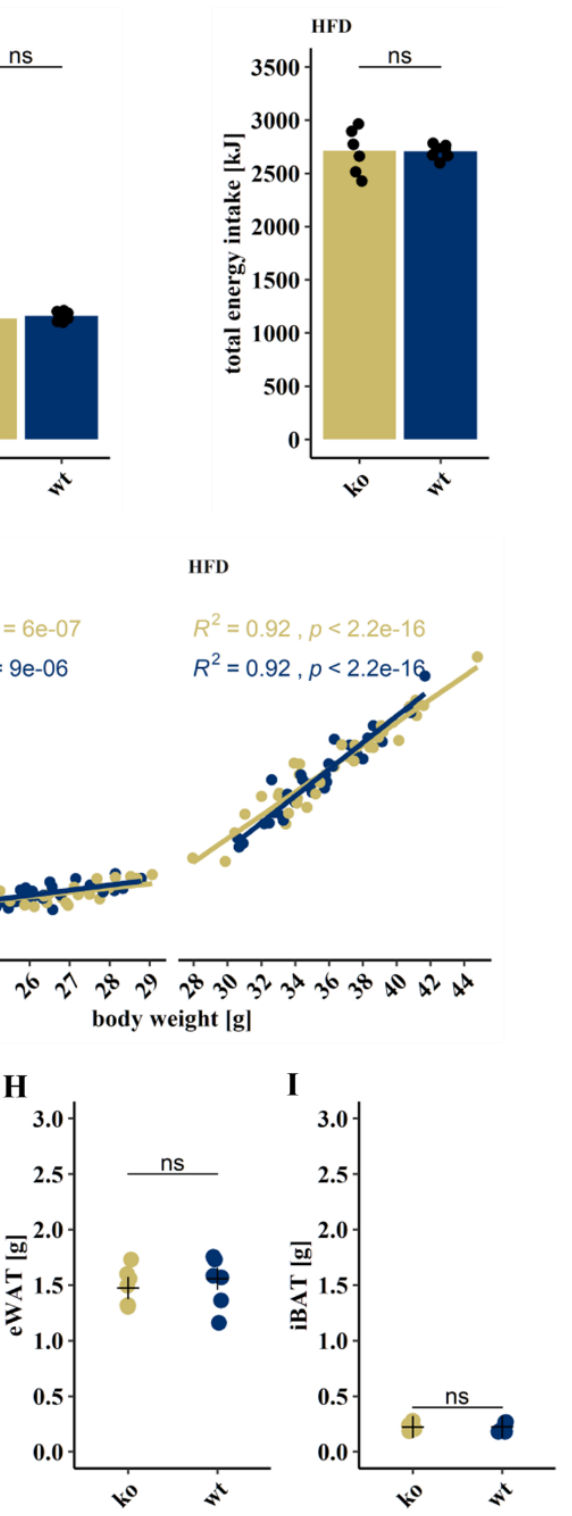

Figure 3: Similar susceptibility to diet induce obesity in UCP1-KO and WT mice.

(A) Body weight of Ucp1-WT (wt, $\mathrm{n}=7$ ) and Ucp1-KO (ko, $\mathrm{n}=7$ ) mice at $30^{\circ} \mathrm{C}$ fed a control (CD) or high-fat diet (HFD). Total energy intake of mice during (B) CD and (C) HFD feeding. (D-E) Pearson correlation coefficient between measurements of (D) lean mass and body weight and (E) fat mass and body weight during CD (left) and HFD (right) feeding. (F) Metabolic efficiency in terms of correlation (Persons correlation coefficient) between cumulative fat mass gain and cumulative energy intake for CD (left panel) and HFD (right panel). Weights of dissected (G) inguinal white adipose tissue (iWAT), (H) epididymal white adipose tissue (eWAT) and (I) interscapular brown adipose tissue (iBAT) at the end of HFD feeding. (B,C,G-I) Student's t-test ns = p-value > 0.05. Group means indicated as (B,C) bars and (G-I) crosses. 
A

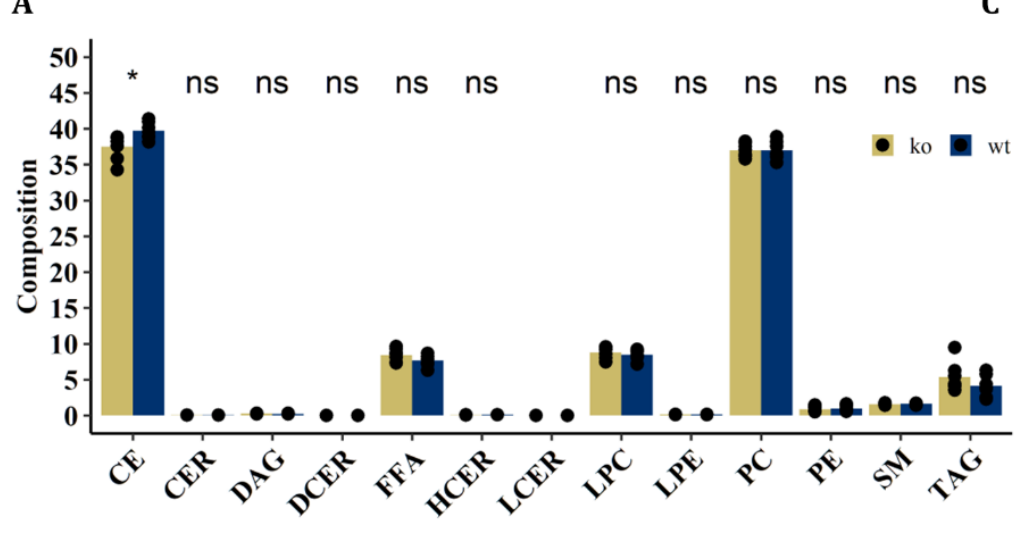

B

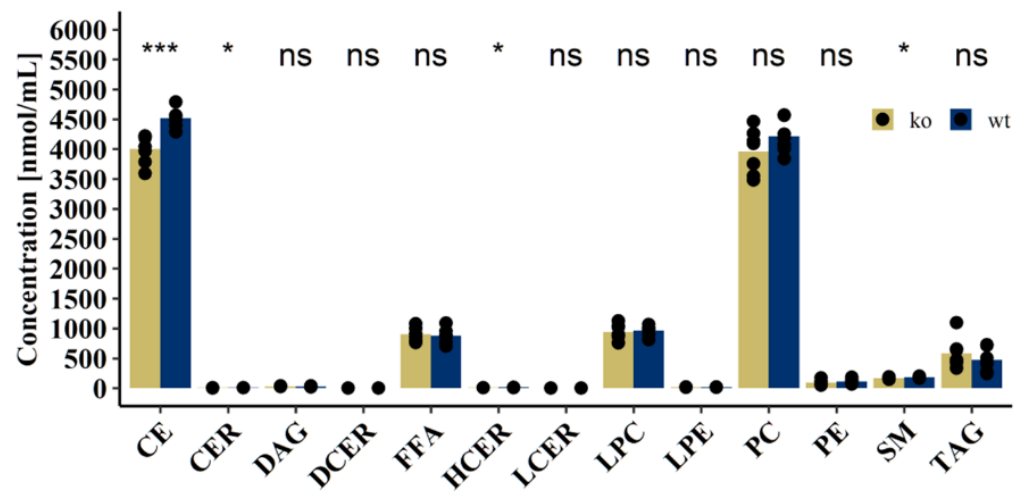

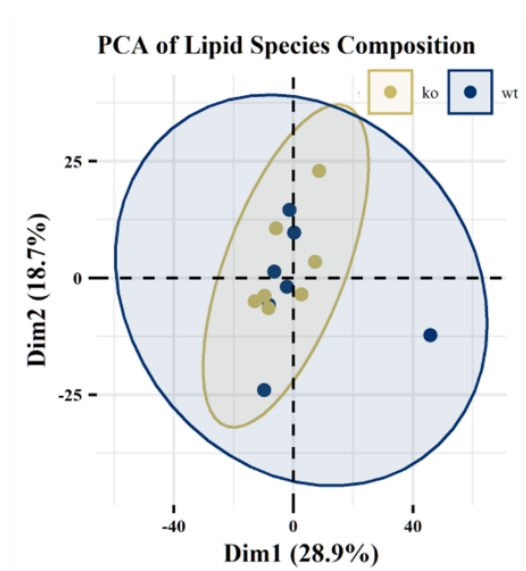

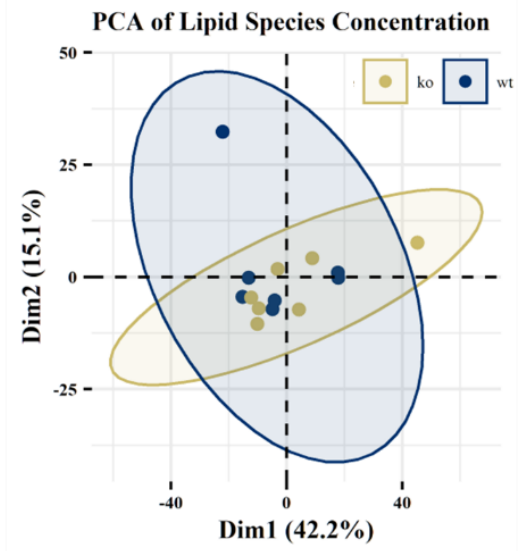

Figure 4: Plasma lipid profiles are comparable between UCP1-KO and UCP1-KO.

(A) Composition and (B) concentration of lipid classes in plasma of UCP1-KO (ko, n =7) and UCP1WT (wt, $\mathrm{n}=7$ ) mice housed at $30^{\circ} \mathrm{C}$ after 8 weeks of high fat diet feeding. Principal component analysis (PCA) of lipid species (C) composition and (D) concentration. Cholesteryl esters (CE), ceramides (CER), diacylglycerols (DAG), dihydroceramides (DCER), free fatty acids (FFA), hexosylceramides (HCER), lactosylceramides (LCER), lysophosphatidylcholines (LPC), lysophosphatidylethanolamines (LPE), phosphatidylcholines (PC), phosphatidylethanolamines (PE), sphingomyelins (SM). triacylglycerols (TAG). (A\&B) Student's t-test ns $=$ p-value $>0.05, *=$ p-value $<0.05, * * *=p$-value $<0.001$. Group means indicated as (A\&B) bars. 
A

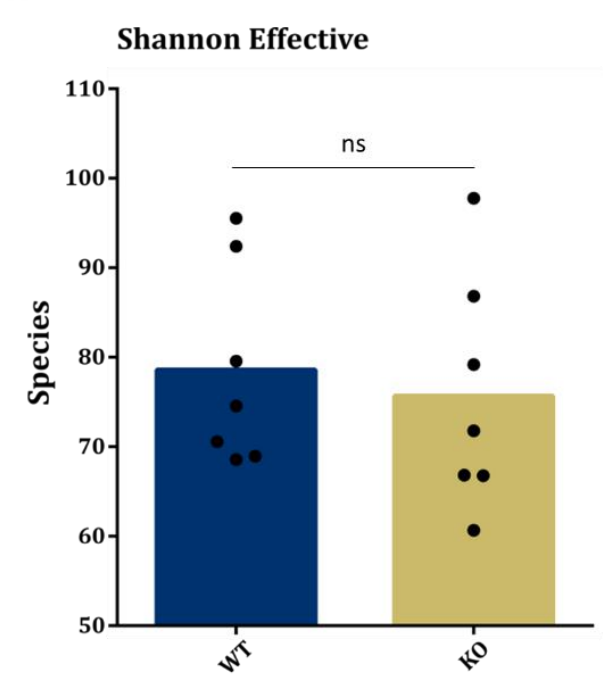

B

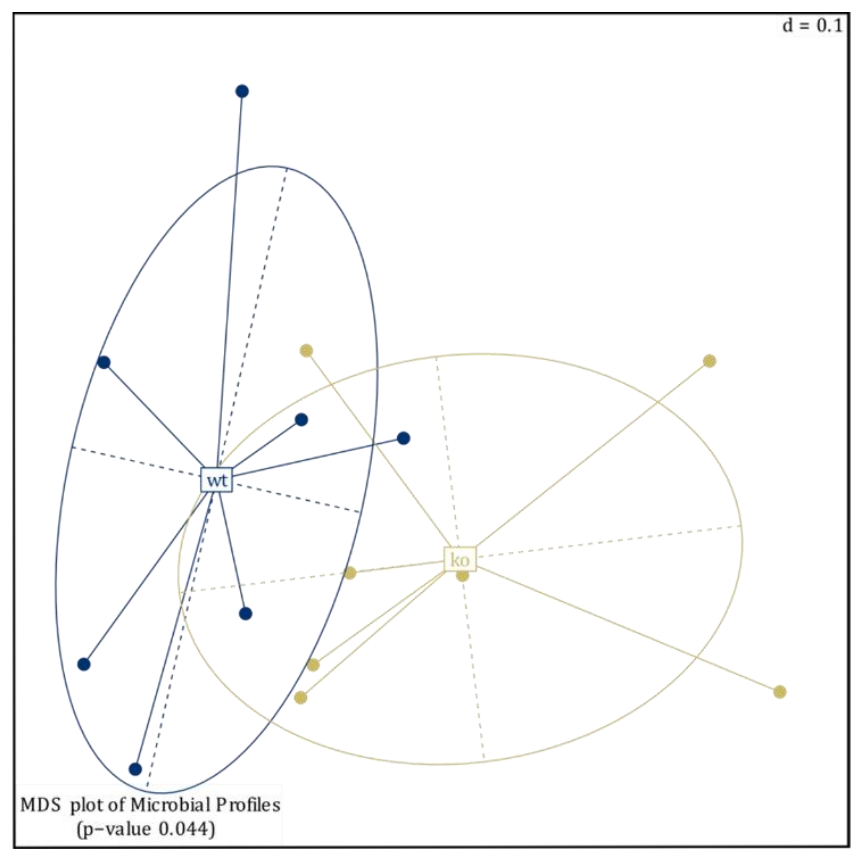

C

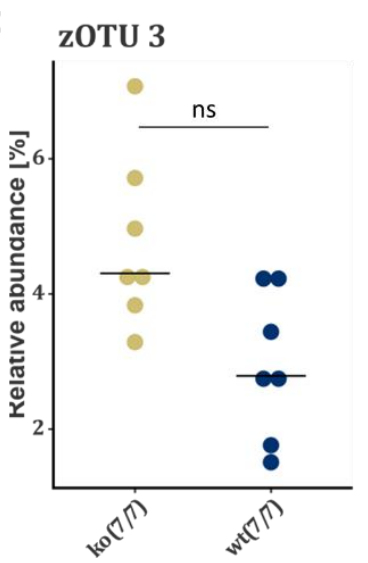

D $\quad$ zOTU 4

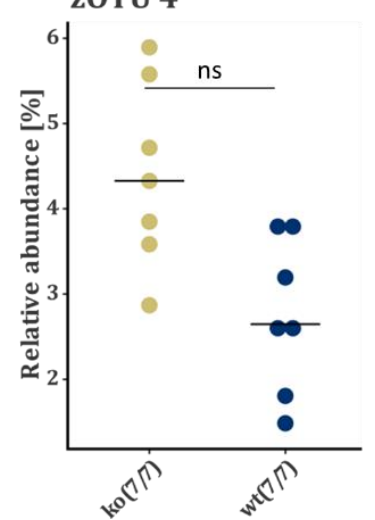

$\mathbf{E}$

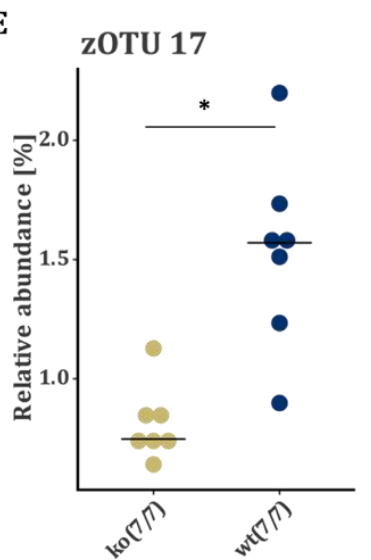

F

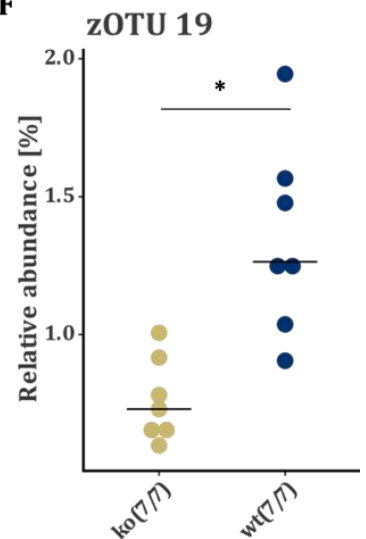

Figure 5: Single cecal microbial genera are associated with the presence of UCP1.

Analysis of cecal microbiome of UCP1-KO (ko, $\mathrm{n}=7$ ) and UCP1-WT (wt, $\mathrm{n}=7$ ) mice housed at $30^{\circ} \mathrm{C}$ after 8 weeks of high fat diet feeding. Comparison of (A) alpha-diversity determined by Shannon effective index and (B) beta-diversity assessed by principal coordinates analysis. (C-F) Relative abundance of zOTU identified by statistically different unadjusted Kruskal-Wallis rank sum test between WT and KO mice. Statistical differences tested by (A) non-parametric Mann-Whitney U test $\mathrm{ns}=\mathrm{p}$-value $>0.05$, (B) Permutational multivariate analysis of variance, (C-F) Kruskal-Wallis rank sum test with the Benjamini \& Hochberg adjustment ns $=$ p-value $>0.05, *=$-value $<0.05$, Group means indicated as (A) bars, (C-F) lines. 
A

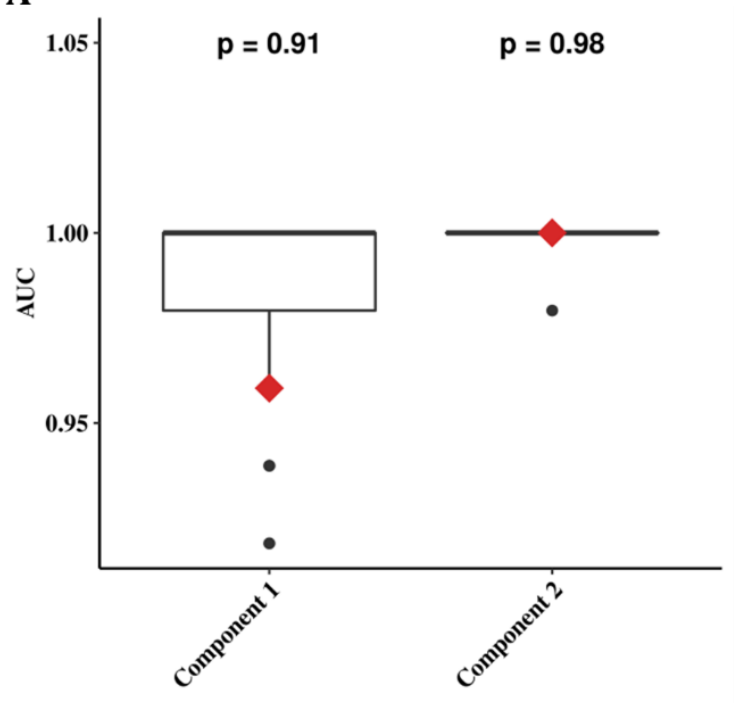

B

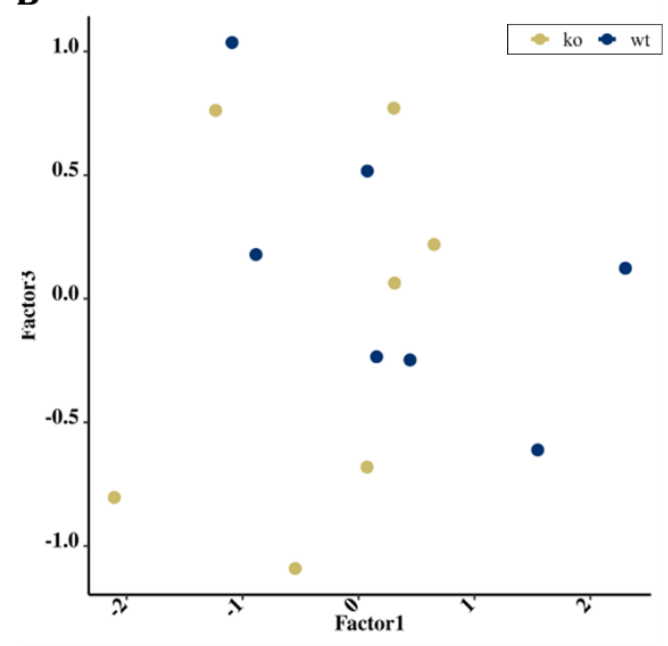

Figure 6: Multi-omics reveal no interaction between microbiome and lipidome explaining differences between UCP1-KO and UCP1-WT mice.

Integrated analysis of the combined lipidome and microbiome data sets. (A) Receiver operating characteristic area under the curve (AUC) of data integration analysis for biomarker discovery using a latent components partial least squares discriminant analysis (DIABLO PLS-DA). Red diamonds indicate results of supervised DIABLO PLSA-DA of the components 1 and 2 (Comp1, Comp2). Box plots indicate results of 1000 randomized DIALBO PLS-DA analyses. P-value $=\#\left(\mathrm{AUC}_{\text {sup }}<\mathrm{AUC}_{\text {ran }}\right) /$ 1000. (B) Visualization of the two factors explaining most of the variance between UCP1-KO and UCP1-WT mice based on multi-omics factor analysis (MOFA). 
A

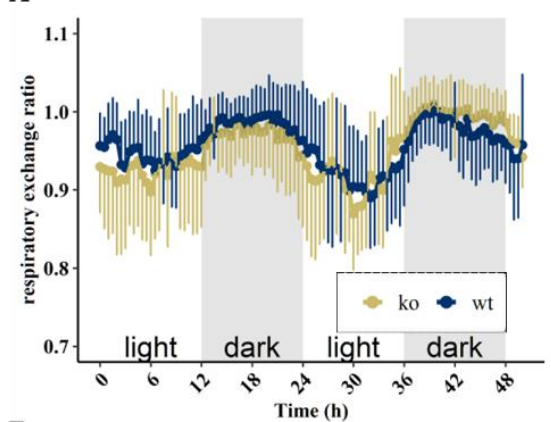

E

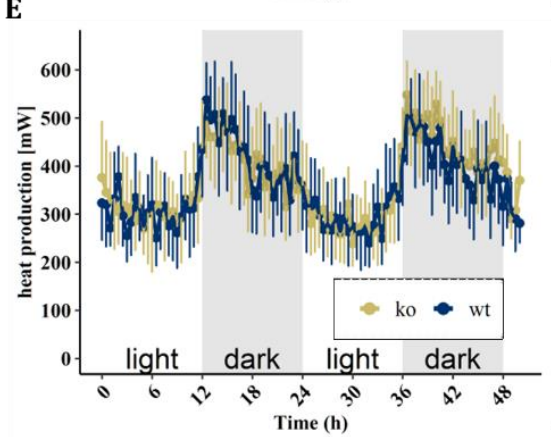

I

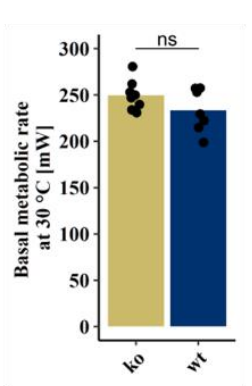

B

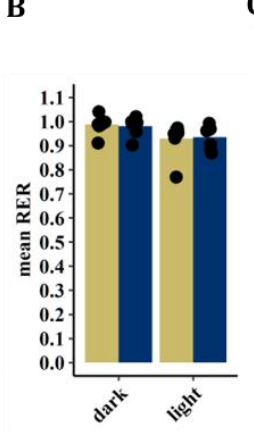

F

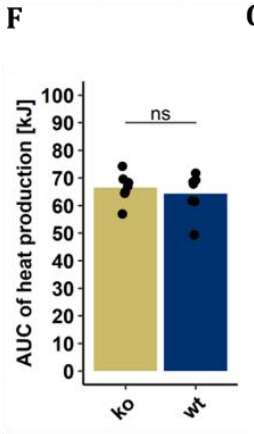

C

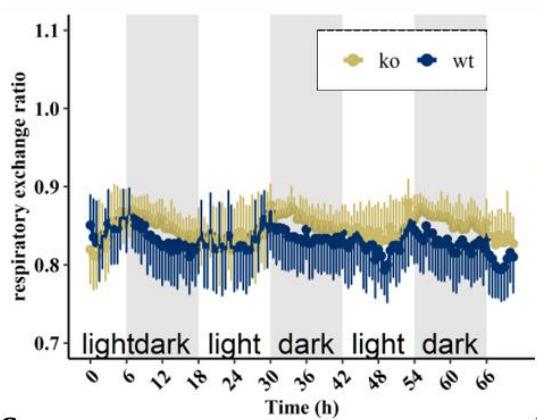

G

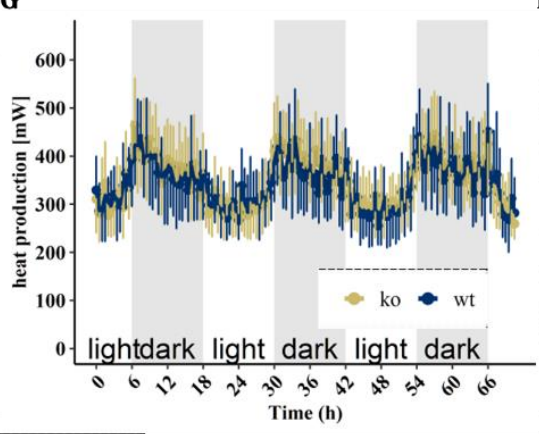

D

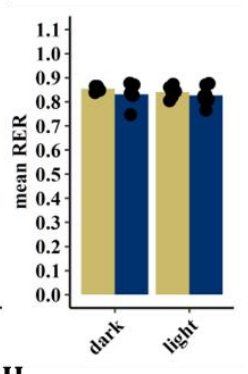

H

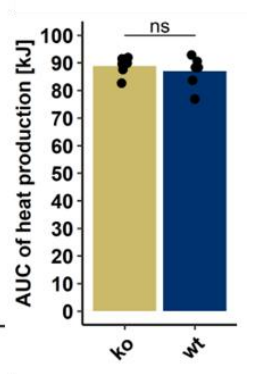

$\mathbf{L}$
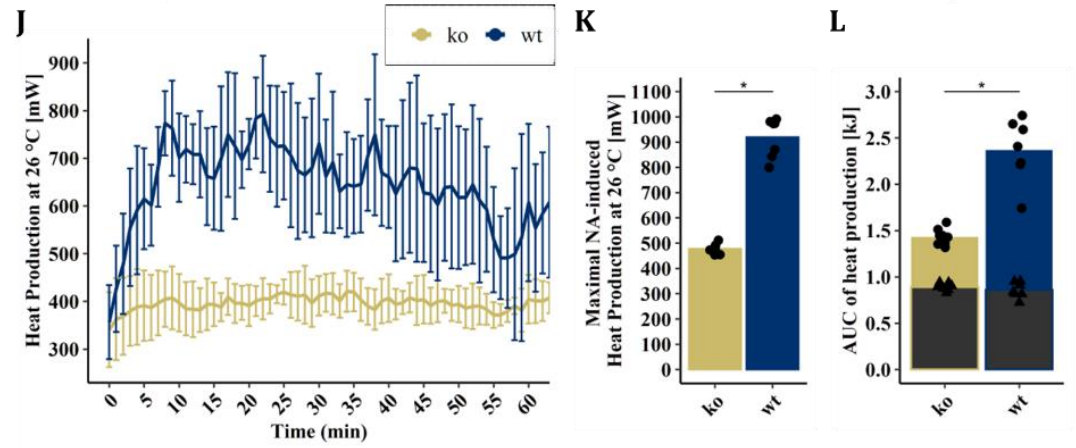

Figure 7: Energy expenditure at thermoneutrality is comparable between Ucp1-KO and UCP1-WT mice.

(A) Respiratory exchange ratio of Ucp1-KO (ko, $\mathrm{n}=7$ ) and Ucp1-WT (wt, $\mathrm{n}=7$ ) mice during CD feeding. (B) mean respiratory exchange ratio (RER) of dark and light phases corresponding to (A). (C) Respiratory exchange ratio of mice during HFD feeding. (D) mean respiratory exchange ratio (RER) of dark and light phases corresponding to $(\mathrm{C})$. (E) Heat production during $\mathrm{CD}$ feeding and (F) the respective area under the curve (AUC). (G) Heat production during HFD feeding and (H) the respective area under the curve (AUC). (I) Mean basal metabolic rate (mean of the four consecutive lowest values after at least $3 \mathrm{~h}$ of fasting) at $30^{\circ} \mathrm{C}$. (J) Heat production curve of mice injected with noradrenalin at 26 ${ }^{\circ} \mathrm{C}$. (K) Maximal heat production during the 80 minutes measurement interval shown in (J). (L) AUC of heat production corresponding to $(\mathrm{J})$. Grey bars and triangles indicating contribution of basal metabolic rate. $(\mathrm{F}, \mathrm{H}, \mathrm{I}, \mathrm{K}, \mathrm{L})$ Students t-test, $\mathrm{ns}=\mathrm{p}>0.5, *=\mathrm{p}<0.5$, bars indicate group means; (A,C,E,G) data represented as means and standard deviation, averaged over a period of $30 \mathrm{~min}$; (J) data represented as means and standard deviation, averaged over a period of $10 \mathrm{~min}$. 
A
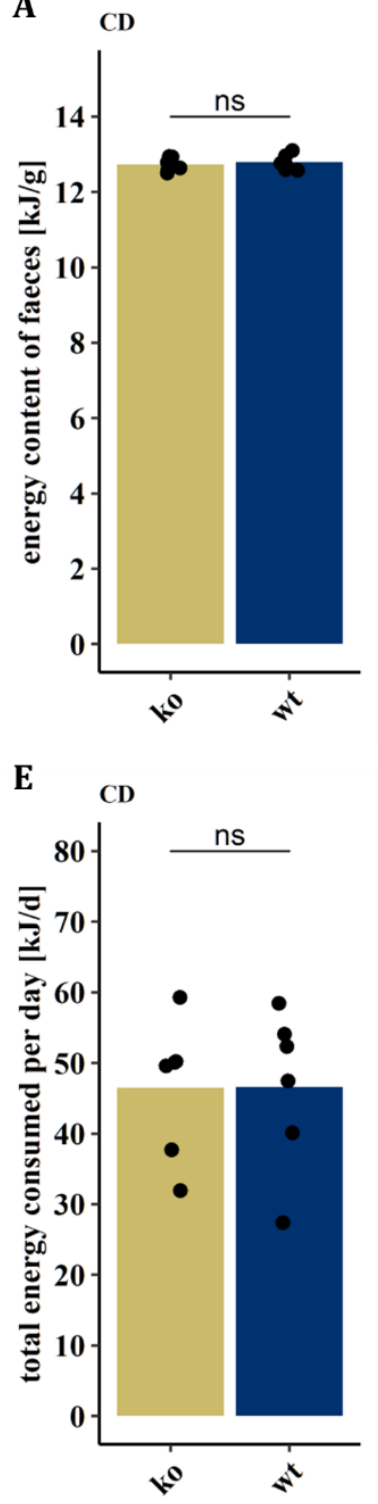

B

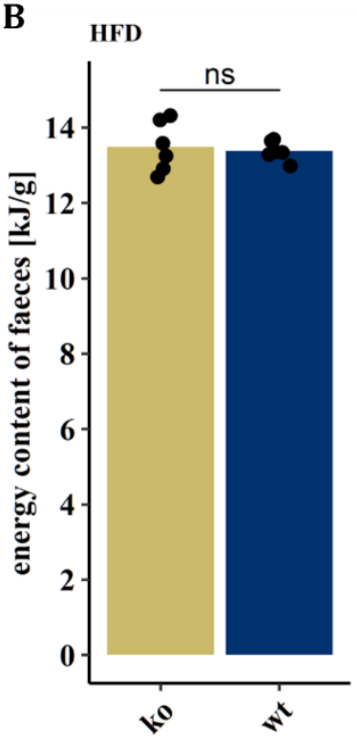

$\mathbf{F}$

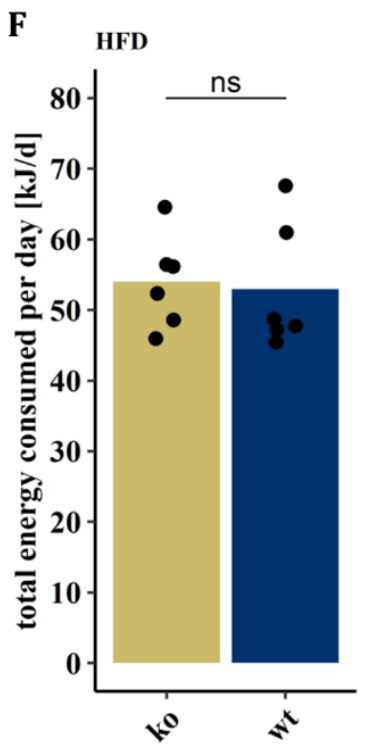

C

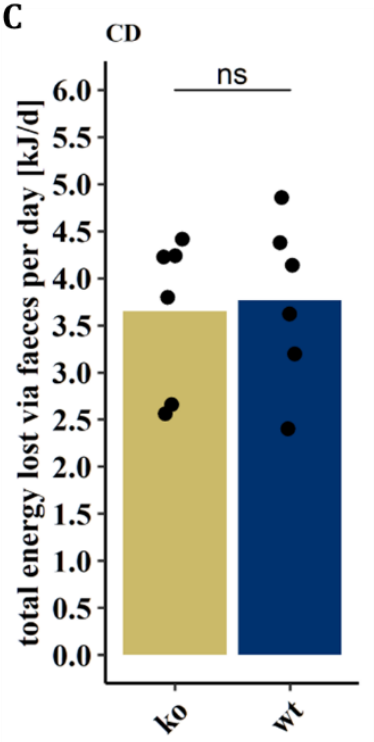

G

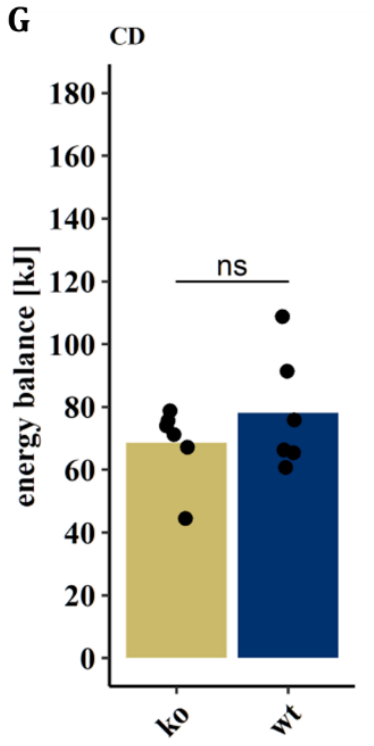

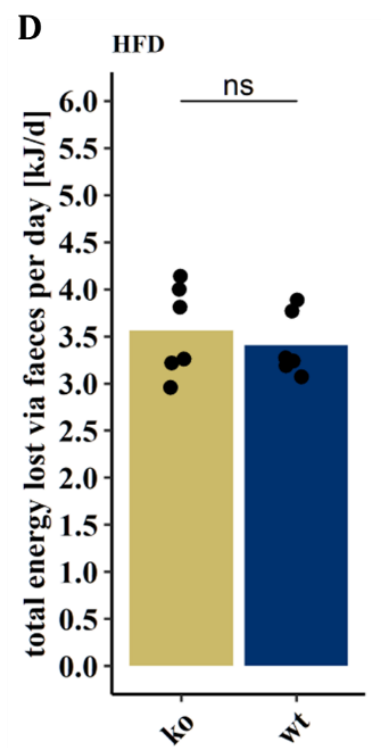

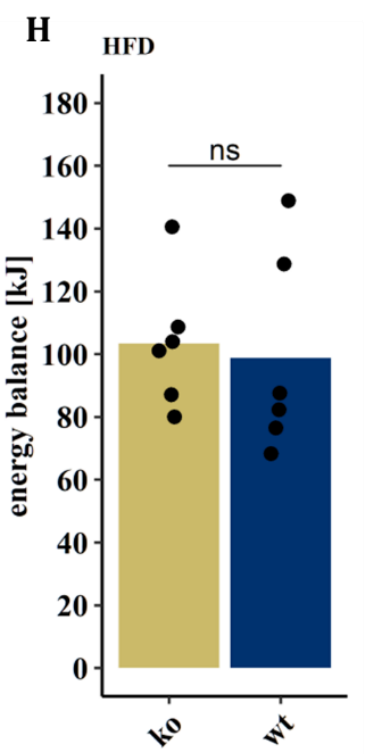

Figure 8: Knockout of Ucp1 does not influence energy balance at thermoneutrality.

Faecal energy content of Ucp1-WT $(n=6)$ and Ucp1-KO $(n=6)$ fed (A) CD or (B) HFD. Total energy lost via faeces of mice fed (C) CD or (D) HFD. Energy consumption of mice fed (E) CD or (F) HFD. Energy balance of mice during (G) CD or (H) HFD feeding. One mouse (wt) was removed as it did not eat during the calorimetry session. Another one (ko) was removed from the analysis as the faecal samples did not combust completely. Students t-test, $n s=p>0.5$, bars indicate group means. 


\section{Tables}

Table 1: Offspring genotype distribution of heterozygous breeding pairs.

\begin{tabular}{lllll} 
Genotype & $\mathrm{N}$ & Observed (\%) & Expected (\%) & $\mathrm{P}\left(\mathrm{X}^{2}\right.$ test $)$ \\
\hline UCP1-WT & 69 & 27.6 & 25 & 0.1364 \\
UCP1-HET & 132 & 52.8 & 50 & \\
UCP1-KO & 49 & 19.6 & 25 &
\end{tabular}

